\title{
Chemokine interactome mapping enables tailored intervention in acute and chronic inflammation
}

Citation for published version (APA):

von Hundelshausen, P., Agten, S. M., Eckardt, V., Blanchet, X., Schmitt, M. M., Ippel, H., Neideck, C., Bidzhekov, K., Leberzammer, J., Wichapong, K., Faussner, A., Drechsler, M., Grommes, J., van Geffen, J. P., Li, H., Ortega-Gomez, A., Megens, R. T. A., Naumann, R., Dijkgraaf, I., ... Weber, C. (2017). Chemokine interactome mapping enables tailored intervention in acute and chronic inflammation. Science Translational Medicine, 9(384), [eaah6650]. https://doi.org/10.1126/scitranslmed.aah6650

Document status and date:

Published: 05/04/2017

DOI:

10.1126/scitransImed.aah6650

Document Version:

Publisher's PDF, also known as Version of record

Document license:

Taverne

Please check the document version of this publication:

- A submitted manuscript is the version of the article upon submission and before peer-review. There can be important differences between the submitted version and the official published version of record.

People interested in the research are advised to contact the author for the final version of the publication, or visit the DOI to the publisher's website.

- The final author version and the galley proof are versions of the publication after peer review.

- The final published version features the final layout of the paper including the volume, issue and page numbers.

Link to publication

\footnotetext{
General rights rights.

- You may freely distribute the URL identifying the publication in the public portal. please follow below link for the End User Agreement:

www.umlib.nl/taverne-license

Take down policy

If you believe that this document breaches copyright please contact us at:

repository@maastrichtuniversity.nl

providing details and we will investigate your claim.
}

Copyright and moral rights for the publications made accessible in the public portal are retained by the authors and/or other copyright owners and it is a condition of accessing publications that users recognise and abide by the legal requirements associated with these

- Users may download and print one copy of any publication from the public portal for the purpose of private study or research.

- You may not further distribute the material or use it for any profit-making activity or commercial gain

If the publication is distributed under the terms of Article $25 \mathrm{fa}$ of the Dutch Copyright Act, indicated by the "Taverne" license above, 


\title{
Chemokine interactome mapping enables tailored intervention in acute and chronic inflammation
}

\author{
Philipp von Hundelshausen, ${ }^{1,2}$ Stijn M. Agten, ${ }^{3}{ }^{*}$ Veit Eckardt, ${ }^{1 *}$ Xavier Blanchet, $^{1}{ }^{*}$ \\ Martin M. Schmitt, ${ }^{1 *}$ Hans Ippel, ${ }^{3 *}$ Carlos Neideck, ${ }^{1 *}$ Kiril Bidzhekov, $^{1}{ }^{*}$ Julian Leberzammer, ${ }^{1}$ \\ Kanin Wichapong, ${ }^{3}$ Alexander Faussner, ${ }^{1}$ Maik Drechsler, ${ }^{1}$ Jochen Grommes, ${ }^{4}$ \\ Johanna P. van Geffen, ${ }^{3}$ He Li, ${ }^{1}$ Almudena Ortega-Gomez, ${ }^{1}$ Remco T. A. Megens, ${ }^{1}$ \\ Ronald Naumann, ${ }^{5}$ Ingrid Dijkgraaf, ${ }^{3}$ Gerry A. F. Nicolaes, ${ }^{3}$ Yvonne Döring, ${ }^{1,2}$ Oliver Soehnlein, ${ }^{1,2,6}$ \\ Esther Lutgens, ${ }^{1,2,7}$ Johan W. M. Heemskerk, ${ }^{2}$ Rory R. Koenen, ${ }^{1,3}$ Kevin H. Mayo, ${ }^{3,8}$ \\ Tilman M. Hackeng, ${ }^{3}$ Christian Weber ${ }^{1,2,3+}$
}

\begin{abstract}
Chemokines orchestrate leukocyte trafficking and function in health and disease. Heterophilic interactions between chemokines in a given microenvironment may amplify, inhibit, or modulate their activity; however, a systematic evaluation of the chemokine interactome has not been performed. We used immunoligand blotting and surface plasmon resonance to obtain a comprehensive map of chemokine-chemokine interactions and to confirm their specificity. Structure-function analyses revealed that chemokine activity can be enhanced by CC-type heterodimers but inhibited by CXC-type heterodimers. Functional synergism was achieved through receptor heteromerization induced by CCL5-CCL17 or receptor retention at the cell surface via auxiliary proteoglycan binding of CCL5-CXCL4. In contrast, inhibitory activity relied on conformational changes (in CXCL12), affecting receptor signaling. Obligate CC-type heterodimers showed high efficacy and potency and drove acute lung injury and atherosclerosis, processes abrogated by specific CCL5-derived peptide inhibitors or knock-in of an interaction-deficient CXCL4 variant. Atheroprotective effects of CCL17 deficiency were phenocopied by a CCL5-derived peptide disrupting CCL5-CCL17 heterodimers, whereas a CCL5 $\alpha$-helix peptide mimicked inhibitory effects on CXCL12-driven platelet aggregation. Thus, formation of specific chemokine heterodimers differentially dictates functional activity and can be exploited for therapeutic targeting.
\end{abstract}

\section{INTRODUCTION}

Chemokines regulate leukocyte activation and coordinate their trafficking to sites of inflammation or during immune surveillance. The $\mathrm{G}$ protein-coupled receptor (GPCR) binding and function of chemokines are governed by their interaction with cell surface proteoglycans, oligomer formation, naturally occurring antagonists, and proteolytic processing $(1,2)$. Recent studies unveiled that heteromeric interactions between chemokines modify their biological activities and provide structural insight into underlying mechanisms. Consequently, we hypothesized that a functional chemokine interactome composed of pairwise heteromeric interactions could inform how signals conferred by individual chemokines can be integrated to control leukocyte responses $(3,4)$.

Many chemokines form homodimers or oligomers based on two modes to support interfaces: CC-type or CXC-type (5). Dimerization in a CC-type occurs via interaction of the flexible $\mathrm{N}$ termini to form a two-stranded antiparallel $\beta$-sheet, whereas in a CXC-type, chemokines interact by antiparallel extension of preformed $\beta$-strands (5). In addition, their structural similarity allows chemokines to form unique heterodimers to shape the overall signaling response of their

\footnotetext{
'Institute for Cardiovascular Prevention (IPEK), Ludwig-Maximilians-Universität München, Munich, Germany. ${ }^{2}$ German Center for Cardiovascular Research (DZHK), Partner Site Munich Heart Alliance, Munich, Germany. ${ }^{3}$ Cardiovascular Research Institute Maastricht, Maastricht University, Maastricht, Netherlands. ${ }^{4}$ Department of Vascular Surgery, RWTH Aachen University, Aachen, Germany. ${ }^{5}$ Max Planck Institute of Molecular Cell Biology and Genetics, Dresden, Germany. ${ }^{6}$ Department of Physiology and Pharmacology, Karolinksa Institutet, Stockholm, Sweden. ${ }^{7}$ Department of Medical Biochemistry, AMC, Amsterdam, Netherlands. ${ }^{8}$ Department of Biochemistry, Molecular Biology and Biophysics, University of Minnesota, Minneapolis, MN, USA.

*These are co-second authors.

tCorresponding author. Email: chweber@med.Imu.de
}

receptors, and homo- and heteromerization of some chemokines are linked to glycosaminoglycan (GAG) binding (4-7). Molecular dynamics simulation (MDS) for selected chemokine homo- and heterodimer pairs predicts that association of free energies depend upon the particular chemokine combination and dimer-type (CC or CXC), some of which are energetically favored over others (8). MDS suggests that CC chemokines preferably engage in CC-type interactions and that $\mathrm{CXC}$ chemokines favor a CXC-type, whereas both interactions can occur in mixed CC-CXC heteromers. Preferred modes of interaction have been validated experimentally for some chemokine pairs but have not been extensively explored or linked to differential functions. Nuclear magnetic resonance (NMR) analyses revealed that CC chemokine receptor 2 (CCR2) ligands form CC-type heteromers and that CCL5 forms a mixed CC-type heteromer with the CXC chemokine CXCL4 $(6,7)$. The CXC-type heterodimer CXCL4-CXCL8 inhibits CXCL8-mediated endothelial cell activation and proliferation binding CXCR2 less strongly than CXCL8 alone $(9,10)$. Peptidemediated disruption of the CCL5-CXCL4 heteromer revealed that this interaction enhances CCL5-mediated leukocyte recruitment, acute lung injury, and atherosclerosis in mouse models $(7,11)$; however, other heteromers have not been evaluated in disease models.

Among the interactions between platelet-derived chemokines identified by mass spectrometry, MDS revealed that CXCL4 can undergo conformational changes to align its $\alpha$-helix with that of CXCL12 to form a CXC-type dimer (12). Without providing clues toward the type of interaction, screening for synergistic effects on leukocytes showed that combinations of CCR7 agonists with some nonagonist chemokines led to formation of heteromers that enhance $\mathrm{T}$ cell migration and CCR2 ligand activity in monocytes $(13,14)$. Such evidence of functional synergy implied that heteromers may act as amplifiers in 
chemokine-rich tissues; however, a systematic and in-depth approach to map interactions between chemokines and to verify their functional specificity has not been undertaken. Here, we have established the chemokine interactome comprising all heterophilic chemokine-chemokine interactions. We further show that CC-type heterodimers mediate functional synergism, whereas CXC-type heterodimers cause inhibitory effects, both of which can be targeted by specific peptides in models of inflammation and atherosclerosis.

\section{RESULTS}

\section{Mapping the chemokine interactome}

Unbiased bidirectional immunoblot chemokine screening, in which one partner was immobilized on a nitrocellulose membrane and the other remained in solution (fig. S1), identified heteromeric interactions between pairwise combinations of all known human chemokines. Interactions were considered positive when they exceeded a densitometry threshold of $5 \%$ (on average) relative to positive controls in either direction (Fig. 1A and table S1). The full interactome matrix revealed hotspots of heteromeric interactions (mostly for inflammatory pairs) and large areas devoid of interactions (Fig. 1A). Neither CC chemokines that adopt unusual polymeric or unique monomer states (CCL3, CCL4, and CCL18) $(15,16)$ nor transmembrane chemokines (CX3CL1 and CXCL16) formed heteromers. With the exception of CCR7 ligands, only a few nonmucosal homeostatic chemokines or plasma chemokines activated by $\mathrm{N}$-terminal cleavage (1) engaged in interactions.

In pursuit of disease-specific therapeutic targets, we focused on chemokines involved in atherosclerosis. CCL2 interacted with CCL5, CXCL8, and other CCR2 ligands (Fig. 1A). The atherogenic CCR1/3/5 agonist CCL5 was the most promiscuous chemokine, forming heteromers with 24 partners (Fig. 1A). Various CXCR2 and CXCR3 ligands (2) interacted with each other and with CCL2, CCL5, or CCL11. We did not consider CCR7 ligands because of their inconclusive role in atherosclerosis models (17). The atypical chemokine CXCL4 interacted with CXCL12 and many atherogenic chemokines. CXCL4L1, a CXCL4 variant that differs at three C-terminal residues $(18,19)$, primarily interacted with CCL19/21/25/27 and CXCL12 but not with CCL5, thus displaying a more homeostatic profile. An explanation as to why CXCL4L1 does not interact with CCL5 is that the changes in the C-terminal helix lead to a larger angle formed with the central $\beta$-sheet and exposure of residues within the core that are usually covered by the helix (19). Because heterodimer formation of CCL5-CXCL4 requires residues from the $\beta$-sheet of CXCL4 (7), this alteration may critically interfere with the binding of CCL5.

The multiple interactions observed for CCL5 and CXCL4 were validated by surface plasmon resonance (SPR), which also confirmed the interactions of atheroprotective CXCL12 with CCL5 or CXCL4 and a more selective interaction pattern of CCL17 (Fig. 1, B to E). Thresholds for SPR and densitometry were defined so as to minimize the number of nonvalidated interactions. In addition to observing positive signals in ligand blots and/or in previous assays, interactions were considered bona fide when they passed these thresholds or had good affinity [dissociation constant $\left(K_{\mathrm{d}}\right)<10^{-6} \mathrm{M}$ ] despite subthreshold SPR responses (Fig. 1, A to E, and table S2).

To elaborate interactions of interest, we performed kinetics analyses, for example, using native CCL5 and CCL5 ${ }^{\mathrm{E} 66 \mathrm{~S}}$ (to obtain equilibrium binding at higher concentrations) on CCL17-conjugated chips (fig. S2). Equilibrium $K_{\mathrm{d}} \mathrm{s}$ revealed that CCL5 and CCL5 ${ }^{\mathrm{E} 66 \mathrm{~S}}$ had equivalent affinity for CCL17 and CXCL4 $\left(K_{\mathrm{d}}<10^{-8} \mathrm{M}\right)$, intermediate affinity for CCL2 and CXCL7 $\left(K_{\mathrm{d}}<10^{-7} \mathrm{M}\right)$, and lower affinity for CXCL12 (table S2). The affinities of CCL5 for CXCL4, $\mathrm{CXCL} 4^{\mathrm{K} 50 \mathrm{E}}$, and the CXCL4 ${ }^{\mathrm{R}>\mathrm{Q}}$ mutant with abolished heparin binding (20) were moderately reduced or similar, whereas CXCL4L1 did not bind CCL5 but retained affinity for CXCL12. Conversely, CCL5 and the mutant CCL5 ${ }^{\mathrm{E} 66 \mathrm{~S}}$ had high affinity for CXCL4, whereas mutation of the basic BBXB cluster (CCL5 ${ }^{44 A A N A 47}$ ) or E26 (CCL5 ${ }^{\mathrm{E} 26 \mathrm{~A}}$ ) resulted in no or impaired binding, identifying residues critical for the interaction. CCL5 and CCL5 ${ }^{\mathrm{E} 66 \mathrm{~S}}$ showed similar affinities for CCL17 and CXCL12. Monomeric CCL5 ${ }^{\mathrm{MT} 7}$ ( $N$-methylated T7) did not bind CXCL4 and bound CCL17 with 17-fold lower affinity, consistent with a role of the CCL5 $\mathrm{N}$ terminus in CC-type interactions. CCL5 ${ }^{\mathrm{E} 26 \mathrm{~A}}$ but not CCL5 ${ }^{44 A A N A 47}$ had high affinity for CCL17, implying a specific role of E26 in binding to CXCL4, with the BBXB motif also involved in binding to CCL17.

\section{Formation of different types of chemokine heterodimers}

We focused our NMR structural studies on CCL5 and partners selected for their range of interactions and inflammatory relevance. To avoid higher-order aggregation (21) and poor spectral characteristics of native CCL5 (22), we used CCL5 ${ }^{\mathrm{E} 66 \mathrm{~S}}$. The CXCL4 ${ }^{\mathrm{K} 50 \mathrm{E}}$ mutant was applied to attenuate CXCL4 tetramer formation and to allay concerns about broadening of NMR spectra due to chemical exchange (23). Heteronuclear single-quantum coherence (HSQC) with ${ }^{15} \mathrm{~N}$-enriched $\mathrm{CCL}^{\mathrm{E} 66 \mathrm{~S}}$ is exemplified by CXCL4 ${ }^{\mathrm{K} 50 \mathrm{E}}$ and CCL17 (Fig. 2, A and $\mathrm{B}$; see full spectra in fig. S3). Substantial resonance broadening and minimal chemical shift $(\Delta \delta)$ changes indicated heteromeric interactions in the intermediate exchange regime. Changes in resonance intensity ( $\Delta$ intensity) and $\Delta \delta$ of the CCL $5^{\mathrm{E} 66 \mathrm{~S}}$ monomer were plotted versus its sequence (Fig. 2, C to F). Because CCL5 ${ }^{\mathrm{E} 66 \mathrm{~S}}$ dimer resonances were much less perturbed (fig. S4, A to D), we concluded that heteromeric interactions with CCL5 ${ }^{\mathrm{E} 66 \mathrm{~S}}$ occur between monomers and not dimers. This was supported by comparing $\Delta \delta$ and $\Delta$ intensity changes for native CXCL4 interactions with monomeric and dimeric $\mathrm{CCL}^{\mathrm{E} 66 \mathrm{~S}}$ (fig. S4, E to $\mathrm{H}$ ) and for $\mathrm{CXCL} 4^{\mathrm{K} 50 \mathrm{E}}$ interactions with ${ }^{15} \mathrm{~N}$-enriched wild-type CCL5 at low concentrations (fig. S5). Relative amounts of heterodimer depend on the presence of various homooligomer states and their respective equilibrium constants. Nevertheless, our subsequent results with the obligate CCL $5^{\mathrm{MT} 7}$ monomer and the noninteracting CXCL4L1 variant that show a lack of functional synergy make off-target effects and altered monomer activity unlikely. Moderate changes in $\Delta \delta$ and homodimers suggest that NMR-deduced binding constants for the heterodimer are higher than those obtained by SPR. Such differences in the affinity range between NMR and SPR are to be expected, primarily because NMR studies require the use of lower $\mathrm{pH}$ values and higher protein concentrations than those present under physiological conditions that are better mimicked by SPR using surface-immobilized binding partners.

The type of heterodimer formed (CC or CXC) was elucidated by analyzing spectral changes ( $\Delta \delta$ and $\Delta$ intensity). The most perturbed CCL ${ }^{\text {E66S }}$ monomer resonances belong to residues ( 7 to 14,30 to 33 , and 47 to 50) involved in CC-type dimers (Fig. 2, C to F, and fig. S4, E and F). In both heteropairs, residues 7 to 11 at the primary CC-type dimer interface displayed relatively large changes. In CCL5 ${ }^{\mathrm{E} 66 \mathrm{~S}}$, residues 47 to 50 (corresponding to $\beta$-strand 3 ) were more perturbed by interactions with CCL17, whereas residues 30 to 33 were more affected by interactions with CXCL4 ${ }^{\mathrm{K} 50 \mathrm{E}}$ or CXCL4. As compared to CCL17, 
Fig. 1. Mapping of the chemokine interactome. (A) Chemokine-chemokine interactions were detected by bidirectional immunoligand blotting. Known atherogenic chemokines are highlighted in orange, and nonmucosal homeostatic chemokines are shaded in gray. No interaction for nonmucosal homeostatic chemokines (gray square) or all other chemokines (white square) is shown. Black squares indicate that antibody binding indistinguishably detects both immobilized and soluble (complexed) chemokine. Chemokine interactions were considered positive (blue) if the densitometric signal exceeded that of the positive control by $5 \%$ (on average) on either side of the blot ( $n=2$ to 4 independent experiments). Chemokine interactions previously identified and experimentally confirmed by other techniques are indicated in dark blue. (B to E) Binding of soluble chemokines (100 nM) to immobilized CCL5 (B), CXCL4 (C), CXCL12 (D), and CCL17 (E) was assessed by SPR. Mass equivalent response units (RU) were compared after the association phase. SPR thresholds (as indicated by dotted lines) optimally delineating binders (black bars) and nonbinders (white bars) were based on median values derived from all tested interactions of CCL5, CXCL4, and CXCL12 (B to D) or were set at $300 \mathrm{RU}$ by interpolation with immunoblotting for CCL17. Data represent means \pm SEM ( $n=3$ to 5 independent experiments).

smaller spectral effects for CXCL4 indicate slightly weaker interactions with CCL $5^{\mathrm{E} 66 \mathrm{~S}}$, confirming that heterodimer stability depends on residue composition and subunit orientation at the interface (8). Although the coexistence of monomeric, homooligomeric, and heterodimeric conformations and formation of both heterodimer types may occur with varying molar ratios (fig. S6), these two chemokine pairs prefer to form CC-type heterodimers (Fig. 2, G and $\mathrm{H}$ ).

As evidenced by CCL5 ${ }^{\mathrm{E} 66 \mathrm{~S}}$-induced ${ }^{15} \mathrm{~N}$-CXCL12 spectral changes that reflect intermediate exchange, CXCL12 and CCL5 also form heterodimers (fig. S7, $\mathrm{A}$ and $\mathrm{B})$. The most perturbed ${ }^{15} \mathrm{~N}$ CXCL12 resonances belong to residues in $\beta$-strand 1 and the C-terminal helix, which are involved in the CXC-type dimer interface. Combining ${ }^{15} \mathrm{~N}$-CCL5 ${ }^{\text {E66S }}$ and unlabeled CXCL12 was also indicative of an interaction but inconclusive in terms of heterodimer-type formed (fig. S7, C and D). Other chemokines, for example, CXCL10, induced relatively nonspecific and moderate spectral changes that are likely attributable to their propensity for homoaggregation, as seen for CXCL10 tetramers (24), and increased viscosity at
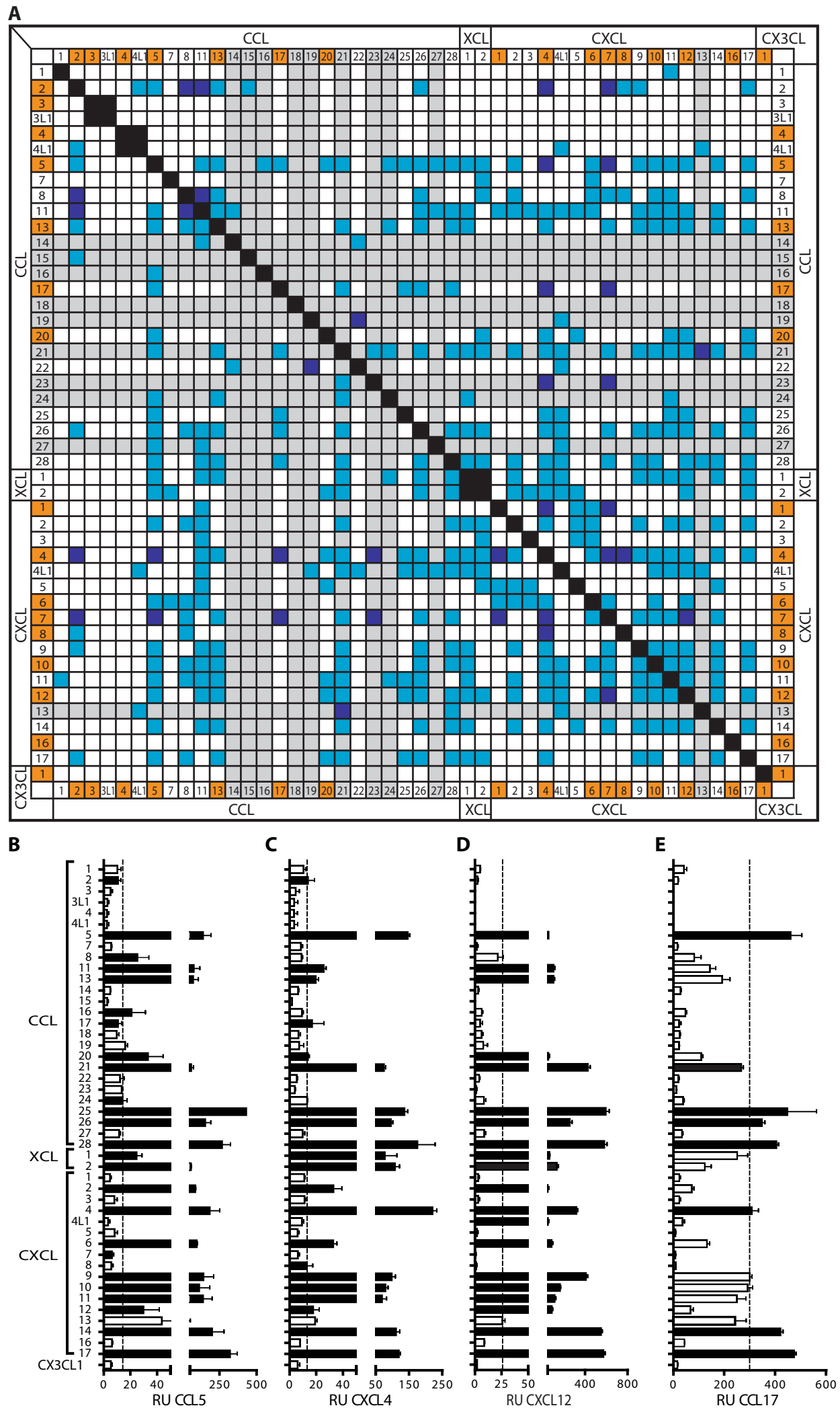

higher concentrations (fig. S7E). Weighted averaging of $\Delta$ intensity changes and MDS-based in silico modeling supported the idea that CXCL12 prefers to form CXC-type interactions with CCL5 and 


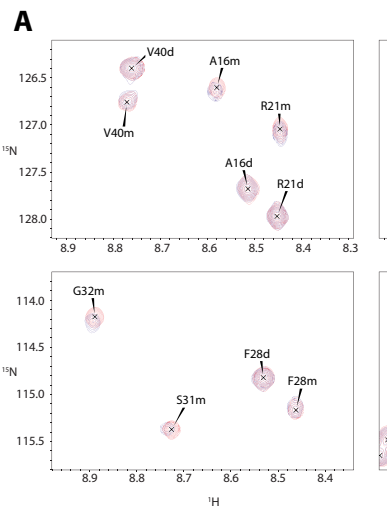

C
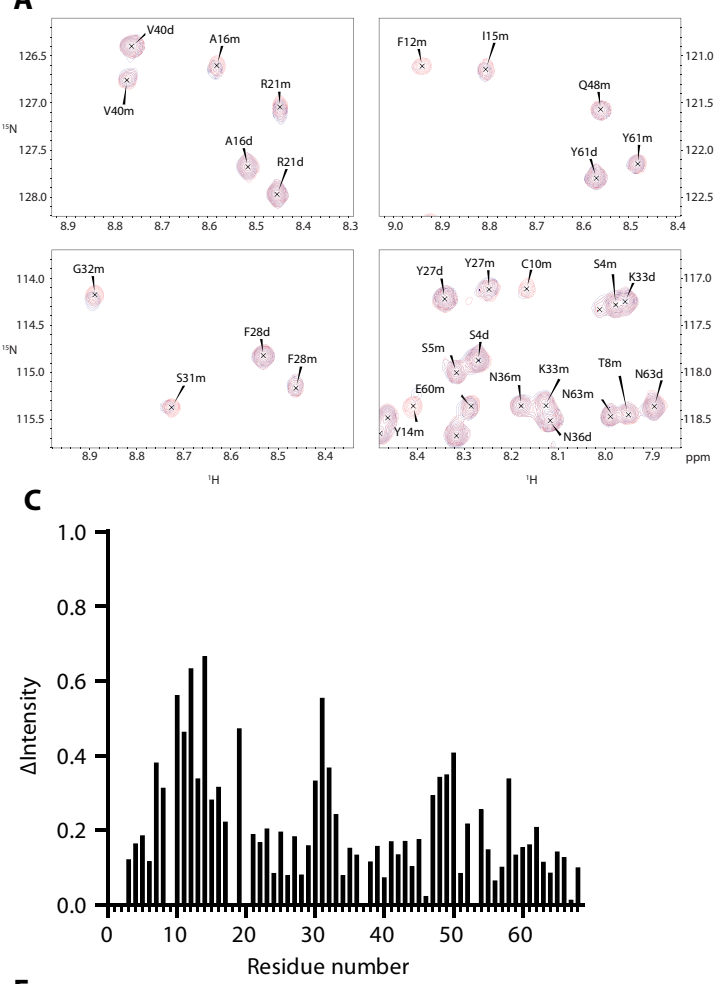

E

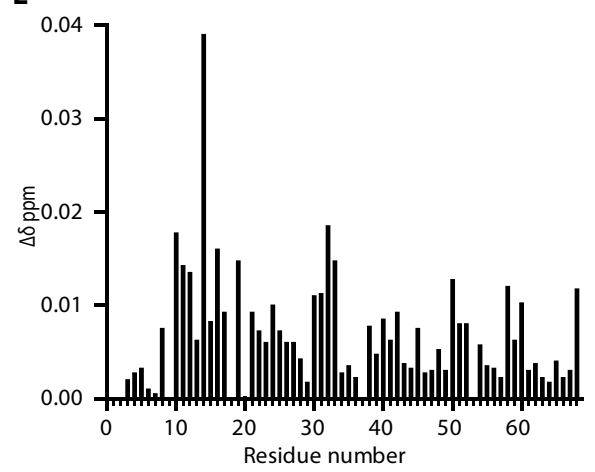

G

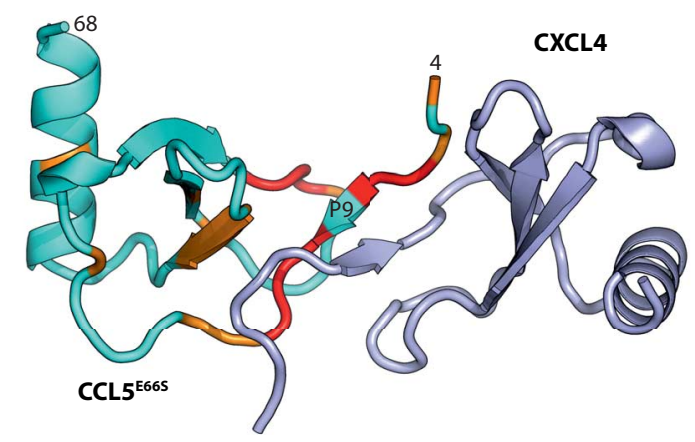

B
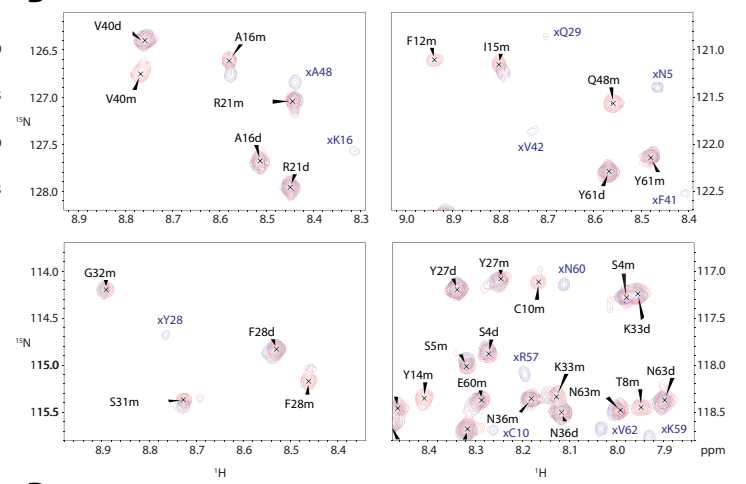

D

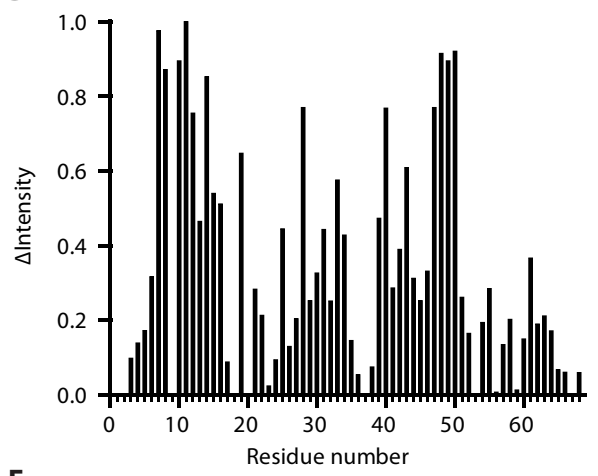

$\mathbf{F}$

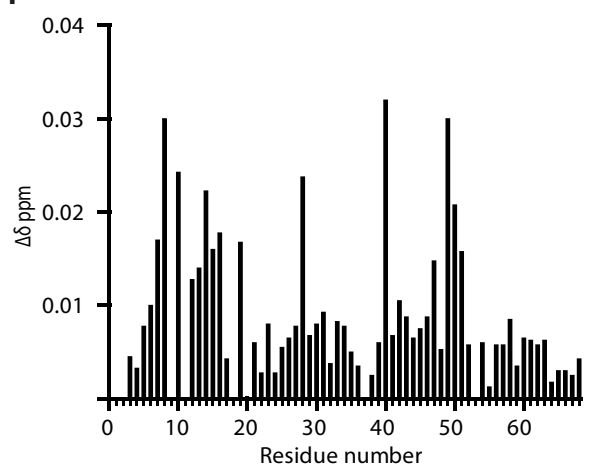

H

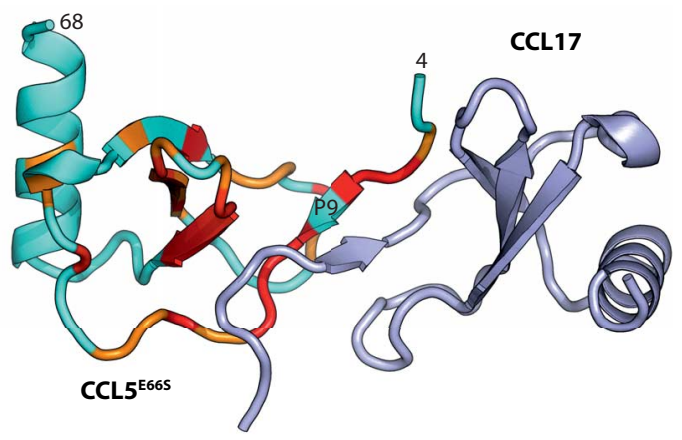

Fig. 2. Characterization of chemokine interactions by NMR spectroscopy. (A to $\mathbf{F}){ }^{1} \mathrm{H}-{ }^{15} \mathrm{~N}$ HSQC spectra were acquired with 128 scans per transient and sweep widths of 16 parts per million (ppm) in the ${ }^{1} \mathrm{H}$ (2000 points) dimension and $22 \mathrm{ppm}$ in the ${ }^{15} \mathrm{~N}$ (200 points) dimension. Expansions are shown for ${ }^{15} \mathrm{~N}$-CCL5 $5^{\mathrm{E} 66 \mathrm{~S}}$, either alone (red) or in the presence of unlabeled CXCL4 (blue) (A) or unlabeled CCL17 (blue) (B); the molar ratios of CCL17/CCL5 ${ }^{\mathrm{E} 665}\left({ }^{1} \mathrm{H} ; 850 \mathrm{MHz}\right.$ ) and CXCL4/CCL5 ${ }^{\mathrm{E} 665}$ ( ${ }^{1} \mathrm{H}$; $700 \mathrm{MHz}$ ) were $33: 1$ $\left(20 \mu \mathrm{M} \mathrm{CCL5}{ }^{\mathrm{E} 665}\right)$ and 20:1 $\left(30 \mu \mathrm{M} \mathrm{CCL5}{ }^{\mathrm{E} 665}\right)$, respectively. Labels indicate monomers $(\mathrm{m})$, dimers $(\mathrm{d})$, and ${ }^{15} \mathrm{~N}$ bleed-through from CCL17 (x). The changes in intensity ( $\Delta$ intensity) and chemical shift $(\Delta \delta)$ for the ${ }^{15} \mathrm{~N}-C C L 5^{\mathrm{E} 665}$ monomer resonances upon binding to CXCL4 (C and E) and CCL17 (D and F) versus those of CCL5 $5^{\mathrm{E} 665}$ are shown. $(\mathbf{G}$ and $\mathbf{H})$ Primary $\mathrm{CCL}^{\mathrm{E} 66 \mathrm{~S}}$ residues binding CXCL4 $(\mathrm{G})$ and $\mathrm{CCL} 17(\mathrm{H})$ heterodimer subunits are indicated on the basis of the native CCL5 homodimer structure (Protein Data Bank 1U4L). The broadest resonances within a single subunit are in red ( $>2$ SD above average), orange (between 1 and 2 SD), and blue ( $<1$ SD). Full-view HSQC spectra are displayed in fig. S3. 
CXCL4, whereas CCL2, CCL17, and CXCL4 favor formation of CC-type heterodimers with CCL5 (table S3).

To exploit our structural models for the design of peptide-based inhibitors, we generated CCL5-derived peptides (CKEY, CAN and VREY), which form part of the heterodimer interfaces with CXCL4, CCL17, and CXCL12, respectively (table S4). Modeled CCL5-CCL17 (CC-type) and CCL5-CXCL12 (CXC-type) heterodimers are illustrated in fig. S8 (A and B). Cyclic CKEY, containing N-loop and $\beta$-sheet 2 with residues K25 to R44 (7), reversed CXCL4-induced changes in $\Delta \delta$ and broadening of ${ }^{15} \mathrm{~N}$-CCL ${ }^{\mathrm{E} 66 \mathrm{~S}}$ monomer, but not homodimer, resonances (fig. S9, A and B). Cyclic CAN comprising CCL5 $\beta$-sheet 3 (residues K33 to N52) interacted with CCL17 (fig. S10) and partially reversed CCL17-induced changes in $\Delta \delta$ and broadening of ${ }^{15} \mathrm{~N}$-CCL5 ${ }^{\mathrm{E} 66 \mathrm{~S}}$ monomer resonances (fig. S11, A and B). Perturbations at the binding interfaces reflect a specific disruption of both CC-type heterodimers. Linear VREY ( $\alpha$-helix residues E54 to S68) mimicked heterodimer interactions of CCL5 with ${ }^{15} \mathrm{~N}$-CXCL12 as similar resonances at the dimer interface were chemically shifted. To improve solubility and conformational stability (25), we generated a four-helix template-assembled synthetic protein (TASP) version of VREY ([VREY] fig. S12A). Changes in $\Delta \delta$ and a structural perturbation plot (fig. S12, B to D) indicated that $[\mathrm{VREY}]^{4}$ interacts with ${ }^{15} \mathrm{~N}$-CXCL12 and attenuates its homo- or heterodimer state, as evidenced by relevant shifts at the interface (fig. S12E).

We validated these findings using SPR, where CKEY inhibited the interaction of CCL5 with CXCL4 but not with CCL17 or CXCL12; this effect was abrogated in the variant $\mathrm{CKEY}^{\mathrm{RE}}$ by substitutions at key residues R44 and E26, crucial for heterodimerization (table S5). Conversely, CAN inhibited the interaction of CCL5 with CCL17 but not with CXCL4 or CXCL12, whereas the N-terminal peptide CCL5 ${ }^{1-33}$ blocked both CC-type interactions of CCL5 with CXCL4 or CCL17 (table S5). Finally, [VREY] ${ }^{4}$ competed with CCL5 for binding to CXCL12 but not to CCL17 or CXCL4 (table S5). These data suggest that chemokine heterodimers can be specifically disrupted by peptides to target their activity.

\section{Differential function and disruption of heterodimers}

To assess the functional effects of chemokine heterodimers, we studied chemotaxis of activated human $\mathrm{T}$ cells, which display a large chemokinereceptor repertoire (fig. S13A) and differentially respond to various chemokines (fig. S13, B to G). Whereas CCL5 plus CCL17 or CXCL4 (CC-type), but not interaction-deficient CXCL4L1, acted synergistically to enhance chemotaxis (Fig. 3, A to D, and fig. S14, A to C), combination with CCL5, CXCL4, or CXCL4L1 (CXC-type) inhibited CXCL12-induced chemotaxis (fig. S14, D to F). Consistent with a lack of specific interactions, CCL5 plus CCL3 or CXCL10 resulted in functionally neutral, that is, neither synergistic nor inhibitory, effects (fig. S14, G and H). Furthermore, combinations preferentially forming CCtype heterodimers (CCL5 plus CXCL4, CCL2, or CCL17) acted synergistically to increase monocyte and/or T cell arrest (Fig. 3, E and F, and fig. S15A). By contrast, combinations forming CXC-type heterodimers, such as CXCL4 and CXCL8 or CCL5 and CXCL12, exerted inhibitory or less than additive effects (fig. S15, B and C). Overall, different types of heterodimeric interactions can have opposite functional consequences, namely, all CC-type interactions identified and functionally tested were synergistic, whereas all CXC-type interactions identified and functionally tested were inhibitory.

In the chemotactic dose-response curve, a left shift demonstrated that CCL5 increased the potency of CCL17 (Fig. 3A), indicating increased affinity or receptor heteromerization. Synergistic effects of CCL5-CCL17 involved both the CCL17 receptor (CCR4) and CCL5 receptors (CCR1 or CCR5), as shown by inhibition with the CCR4 antagonist C021 and the CCL5 receptor antagonist Met-RANTES (fig. S15D). By contrast, CXCL4 increased the efficacy of CCL5 over the entire dose range (Fig. 3B). This effect was blocked by MetRANTES but not by an antibody to the low-affinity CXCL4 receptor CXCR3 (fig. S15E). These data imply that different modes of synergy (affecting potency or efficacy) occur through distinct mechanisms.

Notably, monomeric CCL5 ${ }^{\mathrm{MT} 7}$ did not form strong heterodimers or support functional synergy with CCL17 or CXCL4, whereas the Nterminal peptide CCL $5^{1-33}$ blocked synergistic effects of CCL5 with CXCL4, CCL17, or CCL2, indicating that N-terminal motifs are required for CC-type heterodimer formation and activity (Fig. 3, C, D, G, and H). Consistent with SPR results, CKEY but not CKEY ${ }^{\mathrm{RE}}$ specifically inhibited CCL5-CXCL4 synergy, and CAN specifically inhibited CCL5-CCL17 (Fig. 3, C, D, G, and H, and fig. S15, F and G). Peptide targeting of heterodimer interfaces encompassing $\beta$-strands 2 or 3 with adjacent loops thus confers specificity for functional inhibition. Mimicking the effects of CCL5, [VREY $]^{4}$ but not the other peptides inhibited CXCL12 activity in chemotaxis and arrest (fig. S15, C and H). Along with our NMR data, this indicates that CCL5 and [VREY] ${ }^{4}$ induce structural changes in the CXCL12 dimer, thereby inhibiting CXCL12 activity.

On the basis of modeling (Fig. 2G), we generated a covalently linked CC-type CXCL4-CCL5 heterodimer termed OPRAH (obligate PF4-RANTES heterodimer; Fig. 3I) by introducing an oxime linker between N-terminal residues of CCL5 (T7) and CXCL4 (L8). The efficacy and potency of OPRAH in triggering monocyte arrest and $\mathrm{T}$ cell chemotaxis were greater than those of CCL5 alone or in combination with CXCL4 at concentrations favoring heterodimer formation (Fig. 3J and fig. S15I). By contrast, OPRAH with the $\mathrm{N}$ termini tethered at the first residues (nOPRAH) failed to enhance function (Fig. 3J), showing an importance of freely available $\mathrm{N}$ termini. CKEY did not inhibit the effects of OPRAH (Fig. 3K). An obligate CCL5-CCL17 heterodimer, ORATH (obligate RANTES TARC heterodimer), induced $\mathrm{T}$ cell arrest with higher potency and efficacy than CCL5 and CCL17 combined (fig. S15J), establishing that heterodimers are responsible for the synergistic effects.

To address underlying mechanisms for synergy, we used an in situ proximity ligation assay (26) reporting protein interactions that detect the presence of CCL5-CCL17 heterodimers formed on activated endothelial cells after incubation with both chemokines or with MetRANTES (fig. S16, A and B). Endogenous heterodimers could be detected in mouse lymph nodes (fig. S16, C and D). In dendritic cells (DCs), which express both CCR4 and CCR5, CCL5-CCL17 heterodimers assembled on the cell surface when adding both chemokines, whereas CCR4-CCR5 complexes were constitutively present (Fig. 4A). The increase in CCL5-CCL17 heterodimers was inhibited by CAN (Fig. 4B), revealing that heterodimers can be disrupted by peptides in a cellular context. Notably, the number of CCR4-CCR5 complexes was increased by combining CCL5 and CCL17 but not by either alone (Fig. 4C), as were ligand-receptor cross-interactions (fig. S17, A to D). This effect was impaired by CAN, thus being mediated by CCL5CCL17 heterodimers, and disrupted by CCR5-derived peptides spanning transmembrane 1 and 4 motifs (Fig. 4C), as seen for CCR5 homodimers (27). Peptide-based disruption of CCL5-CCL17 or CCR5-CCR4 impeded synergy in T cell chemotaxis (Fig. 4D and fig. S17E), substantiating a role of ligand-induced receptor heteromer 
A

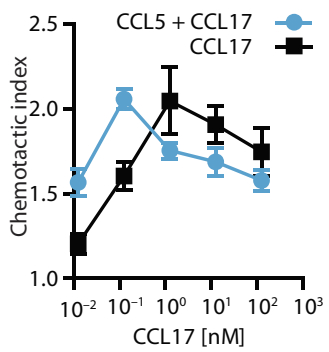

$\mathbf{E}$

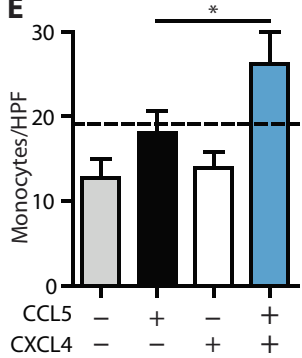

B

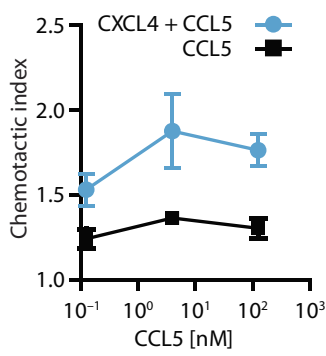

$\mathbf{F}$

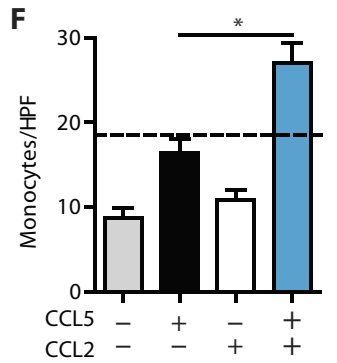

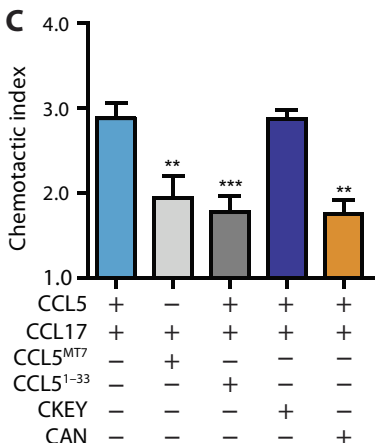

G

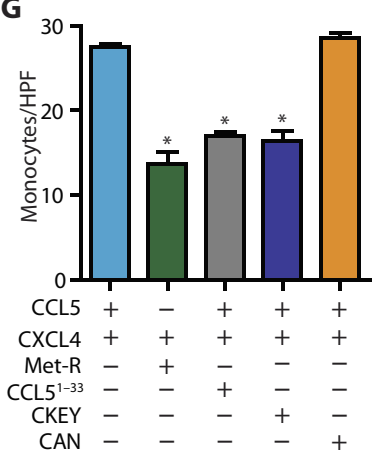

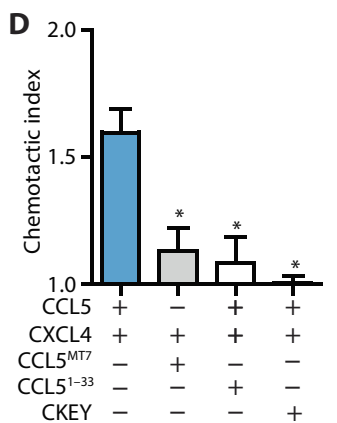

H
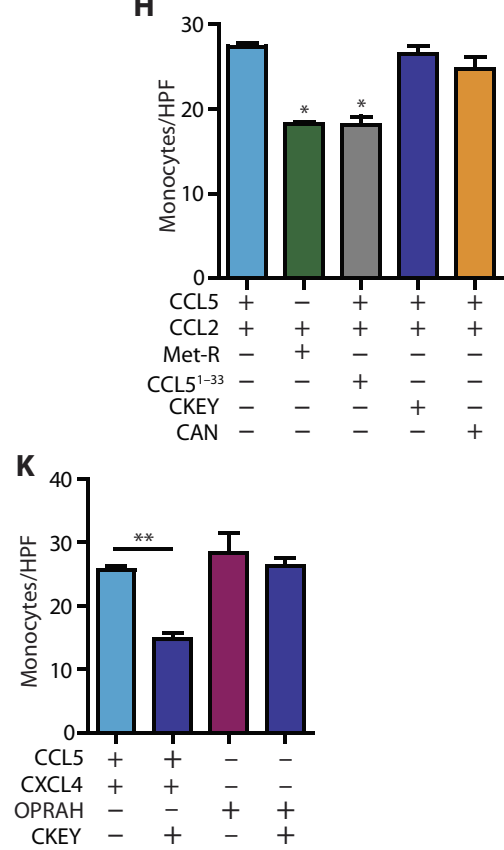

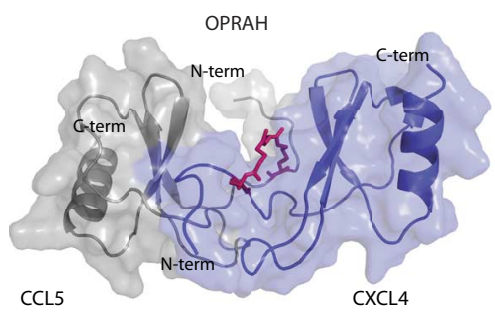

J

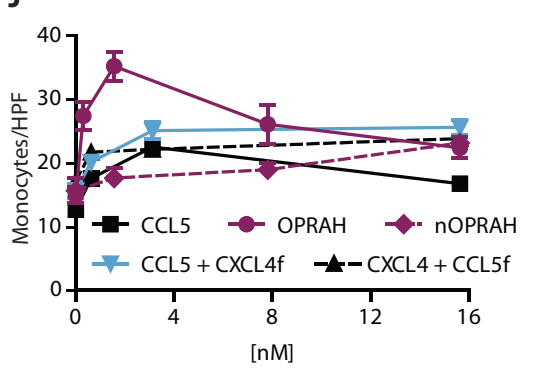

Fig. 3. Differential function and specific inhibition of chemokine combinations. (A to $\mathbf{D}$ ) Transwell filter chemotaxis assays were performed using interleukin-2 (IL-2)-activated and CD3/C28-activated human T cells. The chemotactic index was determined as the ratio of chemokine-induced versus unstimulated migration by counting the cells in the bottom chamber. Combinations of CCL5 (1 nM) plus CCL17 at the indicated concentrations $(n=7)$ (A), CXCL4 (4 nM) plus CCL5 at the indicated concentrations (B) $(n=6), \mathrm{CCL} 5$ or CCL5 ${ }^{\mathrm{MT7}}$ (both $1 \mathrm{nM}$ ) plus CCL17 (0.1 nM; $\left.n=9\right)(C)$, and CCL5 plus CXCL4 (both 4 nM) or obligate platelet factor 4 (PF4)-RANTES heterodimer (OPRAH; $0.4 \mathrm{nM})(n=6)(\mathrm{D})$ were added to the bottom chamber. The CCL5 peptides CCL5 ${ }^{1-33}$ ( $\mathrm{N}$-terminal), CAN (CCL5-CCL17 interface), and CKEY (CCL5CXCL4 interface) were added at 10-fold molar excess. (E to K) Isolated human blood monocytes were perfused over IL-1 $\beta$-activated human aortic endothelial cells (HAoECs) preincubated with chemokines, and numbers of adherent cells per high-power field (HPF) were counted. HAoECs were incubated with CCL5 and/or CXCL4 (40 nM each) (E and $\mathrm{G}$ ) and with CCL5 and/or CCL2 (4 nM each; $n=17)(\mathrm{F}$ and $\mathrm{H})$. Dashed lines indicate virtually calculated additive effects. Monocytes were preincubated with the CCL5 receptor antagonist Met-RANTES (Met-R; $40 \mathrm{nM}$ ), and CCL5 ${ }^{1-33}$, CKEY, or CAN (40 nM each) was combined with CCL5 plus CXCL4 (G) or CCL5 plus CCL2 (H) for pretreating HAoECs ( $n=$ 3). (I) Energy-minimized structure model depicting the CC-type interaction of CCL5 (gray) and CXCL4 (blue) trapped via a covalent oxime linkage (magenta), yielding an obligate PF4-RANTES heterodimer (OPRAH). (J) HAoECs were pretreated with the indicated concentrations of CCL5 plus CXCL4 fixed at 4 nM (CCL5 + CXCL4f), indicated concentrations of CXCL4 plus CCL5 fixed at $4 \mathrm{nM}$ (CXCL4 + CCL5f), or indicated concentrations of OPRAH or nOPRAH (N-terminally tethered OPRAH) ( $n=3$ to 6). (K) HAoECs were pretreated with CCL5 plus CXCL4 or OPRAH (all $4 \mathrm{nM}$ ) with or without CKEY ( $40 \mathrm{nM} ; n=5)$. Data represent means \pm SEM from the indicated numbers of independent experiments. ${ }^{*} P \leq 0.05,{ }^{*} P \leq 0.01,{ }^{* *} P \leq 0.001$ versus chemokine combinations (cyan), as analyzed by one-way analysis of variance (ANOVA) (C, E, and $\mathrm{F}$ ) or Kruskal-Wallis test $(D, G, H$, and $K)$.

complexes. Binding competition assays revealed that the affinity of CCL17 for CCR4 was higher when adding CCL5 but not CXCL1, to promote heterodimer formation (Fig. 4E and fig. S17F). To test whether CCL5-CCL17 can elicit receptor heteromer activity distinct from its monomers, we used GloSensor transfectants expressing CCR4 or CCR4 and CCR5 to assess cyclic adenosine monophosphate (cAMP) signaling. Our data show that CCR4 and CCR5 together mediate the synergistic effects of CCL5-CCL17 on $\mathrm{G}_{\mathrm{i}}$-mediated inhibition of cAMP formation (fig. S17G). In contrast, transfectants expressing CCR1 only efficiently responded to OPRAH (fig. S18A). As for a combination of CCL2 and CCL5 favoring CCR2-CCR5 heterodimerization to enhance cell arrest (28), phosphatidylinositol 3-kinase signaling was also addressed by CCR4-CCR5 heteromers, in that combining CCL5 and CCL17 induced its sustained activation (fig. S18, B and C).

To identify mechanisms underlying CXCL4-mediated synergy, we used the GAG binding-impaired mutant $\mathrm{CXCL4} 4^{\mathrm{R}>\mathrm{Q}}$, which forms heterodimers with CCL5 (table S2). This mutant did not enhance CCL5-induced monocyte arrest, suggesting that GAG binding through 
A
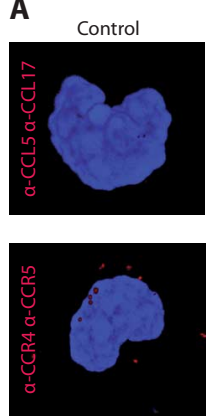

C

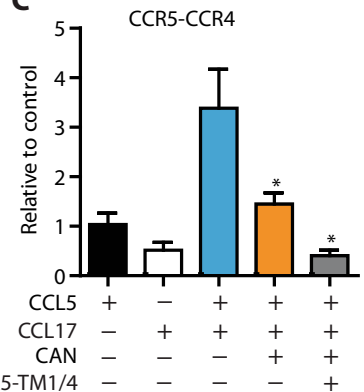

CCL5
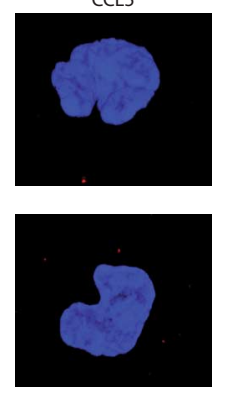

D
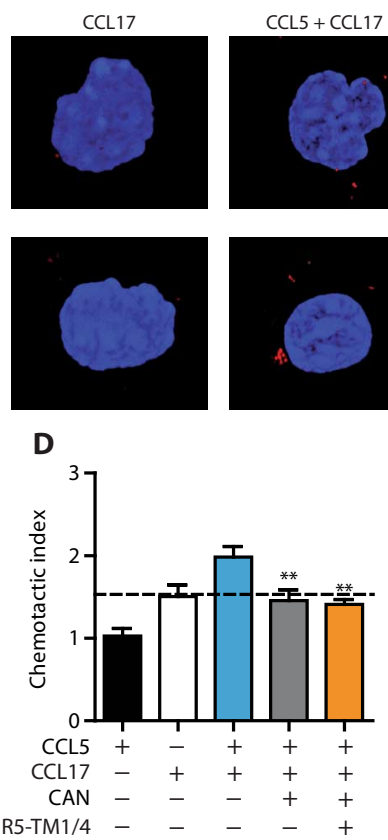

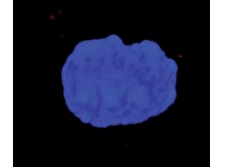

B
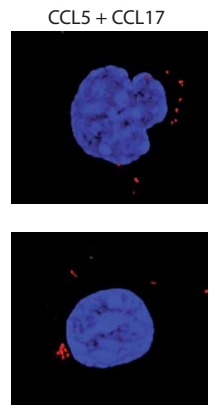

E

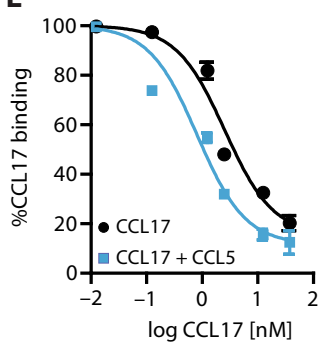

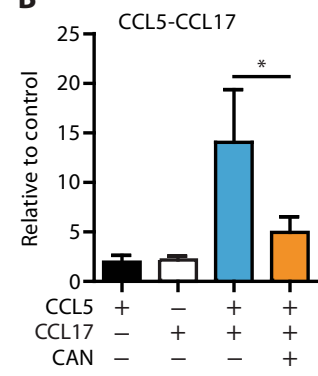

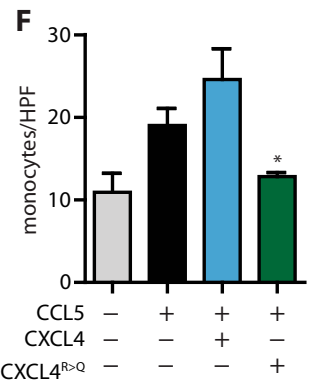

G

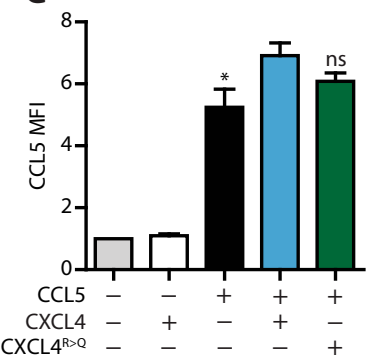

H

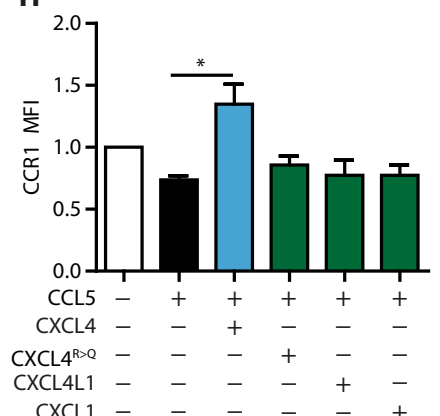

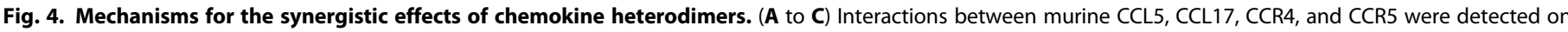

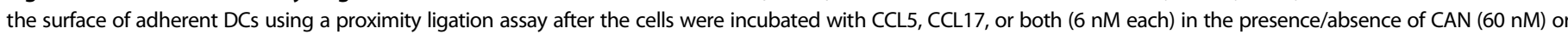

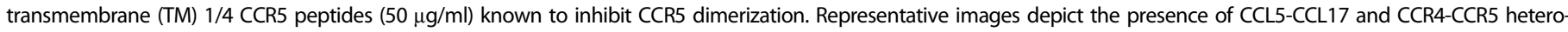

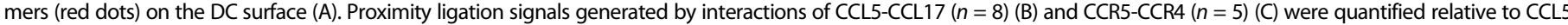

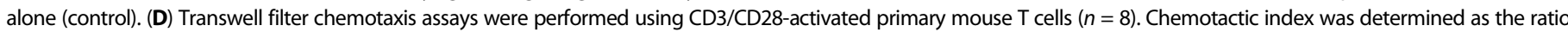

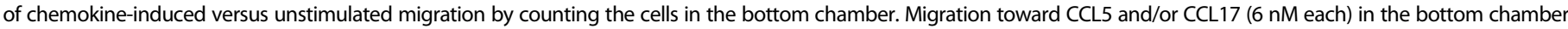

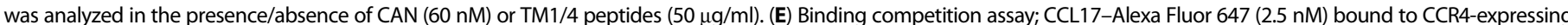

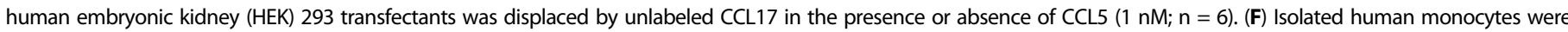

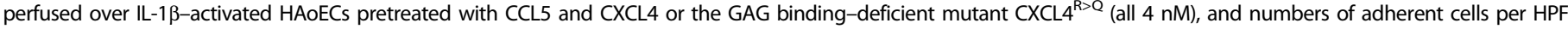

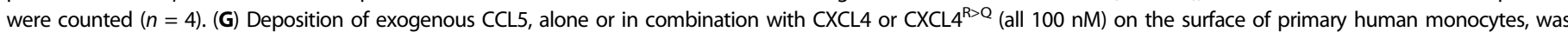

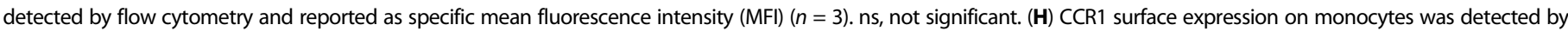

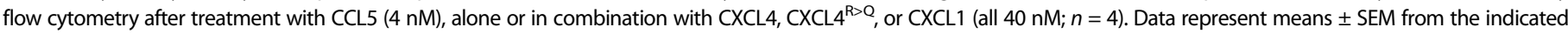

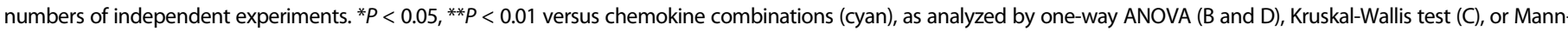
Whitney test ( $F$ and $G$ ).

CXCL4 may improve presentation of CCL5 to its arrest-triggering receptors (Fig. 4F). CXCL4 but not $\mathrm{CXCL} 4^{\mathrm{R}>\mathrm{Q}}$ increased surface binding of CCL5 to monocytes, indicating that this was mediated by GAG binding via CXCL4 (Fig. 4G). As a consequence, CXCL4 but not CXCL4 ${ }^{\mathrm{R}>\mathrm{Q}}$ prevented CCR1 internalization by CCL5 (Fig. $4 \mathrm{H}$ ), without altering the affinity of CCL5 for CCR1 or CCR5 (fig. S18, D and E). This effect was also observed using primary mouse monocytes adherent in carotid arteries perfused ex vivo (fig. S18F). CXCL1 and CXCL4L1, which neither specifically interact with CCL5 nor activate CCR1, but bind to GAGs, did not recapitulate the effect on CCR1 internalization observed with CXCL4, indicating that this is dependent on heterodimer formation with CCL5 (Fig. 4H). SPR on a low-molecular weight heparin-conjugated chip revealed binding of CXCL4L1 but not of CXCL4 ${ }^{\mathrm{R}>\mathrm{Q}}$, and combination with wild-type CXCL4 but not with CXCL4L1 or CXCL4 ${ }^{\mathrm{R}>\mathrm{Q}}$ supported strong binding of CCL5 ${ }^{\mathrm{E} 66 \mathrm{~S}}$ on immobilized heparin (fig. S19A). Such GAG-mediated binding of CCL5containing heterodimers was confirmed by binding of OPRAH to heparin with similar affinity as CCL5 ${ }^{\mathrm{E} 66 \mathrm{~S}}$ (fig. S19, B and C). Thus, the GAG-binding capacity of CCL5-CXCL4 heterodimers limits receptor internalization from the cell surface, where it is retained for continuous 
$\mathrm{G}$ protein signaling, as seen for GPCR families lacking $\beta$-arrestin recruitment for endosomal targeting (29). Experiments using HEK293 transfectants coexpressing C-terminally tagged CCR5-RlucII and enhanced yellow fluorescent protein-tagged arrestin $1 / 2$ to analyze bioluminescence resonance energy transfer events confirmed that the presence of CXCL4 inhibited the CCL5-induced association of arrestin with CCR5 (fig. S19D), reflecting a prolonged signaling cycle.

\section{Specific targeting of heterodimers in inflammatory disease models}

To confirm a role of heterodimer formation in vivo, we used a shortterm model of lipopolysaccharide (LPS)-induced acute lung injury, which relies on platelet chemokines mediating neutrophil recruitment and subsequent extravasation (11). Treatment with CKEY (but not with $\mathrm{CKEY}^{\mathrm{RE}}$ ) dose-dependently reduced infiltration in the lung, as evidenced by lower intravascular, interstitial, bronchoalveolar, and total neutrophil counts (Fig. 5A and fig. S20, A to C). Conversely, reconstitution of mice carrying $\mathrm{Ccl5}^{-/-} \mathrm{Cxcl}^{-/-}$bone marrow (deficient in the heterodimer pair) with OPRAH but not with CCL5 exacerbated lung infiltration, as reflected in higher intravascular neutrophil numbers and a trend toward higher total counts (Fig. 5B and fig. S20D).

To demonstrate the biological relevance of heterodimer interactions, we generated $C x c l 4^{L 1 / L 1}$ mice with a CXCL4 knockout and knockin of the CXCL4L1 variant (which neither exists in mice nor forms heterodimers with CCL5) to study diet-induced atherosclerosis on an $A p o e^{-/-}$background (fig. S21). Notably, Cxcl4 ${ }^{L 1 / L 1}$ mice showed a marked reduction of atherosclerotic lesion size in the aorta and the aortic arch, comparable to that in CXCL4-deficient mice (Fig. 5, C and D, and fig. S22, A and B). Heterozygous $\mathrm{CxCl}^{\mathrm{L1/+}}$ mice displayed an intermediate gene-dosing effect. Lesion size in the aortic root was attenuated in $\mathrm{CxCl}^{-/-}$mice but not in $\mathrm{Cxcl} 4^{\mathrm{L1} / \mathrm{L} 1}$ mice (fig. S22, C and D), implying regional differences unrelated to heterodimerization. Except for lower macrophage content in $C x c l 4^{L 1 / L 1}$ mice, plaque composition (that is, phenotype classification, smooth muscle cells, necrotic core, and T cell content), lipid profiles, body weight, and blood cell counts did not differ among all groups (Fig. 5E, fig. S23, and table S6). Platelet-specific expression of a CXCL4L1 transgene in $A p o e^{-/-}$mice did not alter lesion size, indicating that its effects are not dominant negative but are due to a lack of interaction (Fig. 5F). Treatment with mouse OPRAH restored lesion formation in the aorta including the arch and increased macrophage content in aortic root plaques but did not change other parameters in $\mathrm{Ccl}^{-/-} \mathrm{CxCl}^{-/-} \mathrm{Apoe}^{-/-}$mice and heterozygous $\mathrm{Cxcl} 4^{L 1 / w t} \mathrm{Apoe}{ }^{-/-}$ mice expressing noninteracting CXCL4L1 (Fig. 5, G and H, fig. S24, and table S7).

The atheroprotective homeostasis of regulatory $\mathrm{T}$ cells $\left(\mathrm{T}_{\text {regs }}\right)$ is suppressed by DC-derived CCL17, a phenotype not replicated in Ccr4-deficient mice $(3,30,31)$, possibly implicating other chemokine receptors or heterodimers. We detected CCL5-CCL17 heteromers in aortic root lesions of $A$ poe $e^{-/-}$mice (Fig. 6A), in the intima and adventitia of human coronary arteries with advanced atherosclerosis but not in undiseased vessels or segments (Fig. 6B). Notably, combined interference with CCR4-CCR5 using the CCR5 antagonist DAPTA in CCR4-deficient Apoe ${ }^{-/-}$mice increased $\mathrm{T}_{\text {reg }}$ numbers in the paraaortic lymph nodes and, marginally, in peripheral blood (Fig. 6, C and D). Similar results were obtained by treatment with CAN (for 6 weeks), which also reduced lesion size in the aortic root (Fig. 6, C to F), phenocopying the effects of CCL17 deficiency (30). These findings may be due to limiting CCL17-mediated recruitment of naïve $\mathrm{CD}^{+} \mathrm{T}$ cells, in which $\mathrm{T}_{\text {reg }}$ conversion or maintenance is suppressed via subsequent CCL17-dependent pathways involving other receptors.

To exploit inhibitory interactions exemplified by the CXC-type heterodimer CCL5-CXCL12, we tested whether [VREY] ${ }^{4}$ inhibits CXCL12-depedent platelet aggregation, as relevant to atherothrombosis. Notably, CXCL12 facilitated platelet aggregation as deletion of Cxcl12 in blood of $\mathrm{CreER} \mathrm{R}^{T 2} \mathrm{Cxcl1} 2^{f l f l} \mathrm{Apoe^{- \rho - }}$ mice showed reduced multiparameter platelet activity and thrombus scores ex vivo (Fig. 6, $\mathrm{G}$ and $\mathrm{H})$. Treating $A$ poe $e^{-/-}$mice with $[\mathrm{VREY}]^{4}$ afforded a similar inhibition of platelet activity (Fig. 6, G and H). Likewise, [VREY] ${ }^{4}$ inhibited CXCL12-induced human platelet aggregation and CXCR4 activation in HEK293 cells, likely due to conformational effects on CXCL12 (Fig. 6, I and J).

\section{DISCUSSION}

The comprehensive map of all heteromeric chemokine interactions obtained by immunoligand blotting and SPR contributes to the completion of the binary human protein interactome network (32). We found that inflammatory and nonhomeostatic chemokines, for example, platelet-derived atherogenic CCL5 and CXCL4, favor interactions, whereas homeostatic chemokines are generally less interactive. This may correspond to the need to amplify, fine-tune, and resolve chemokine activity at inflammatory sites through heteromeric interactions, whereas the stand-alone functions of noninteracting chemokines appear sufficient for organogenesis, immune architecture, and surveillance or antimicrobial activity. For example, CXCL4-deficient mice have no overt phenotype and show unaltered immune responses in the absence of challenge, whereas CXCL12 deficiency entails embryonic lethality, and CXCL13 deficiency shows its essential role for lymph node development and natural immunity $(33,34)$.

Structure-function analysis revealed an interesting dichotomous pattern wherein CC-type heterodimers promote synergy and CXCtype heterodimers cause inhibition. The use of obligate heterodimers such as OPRAH provided conclusive proof that CC-type heterodimers mediate functional synergy. Functional inhibition with a CCL5derived N-terminal peptide supports a more general role of the $\mathrm{N}$ terminus in CC-type heterodimer formation and synergy. The spacing and shapes adopted by CC-type homodimers can vary with minimal changes in N-terminal sequence (16). Functional synergy indeed involved flexible $\mathrm{N}$ termini, as illustrated by a tethered version of the obligate heterodimer OPRAH, and could be ascribed to addressing receptor heteromers (increasing potency of CCL5-CCL17) or to auxiliary GAG binding and impaired receptor internalization (increasing efficacy of CCL5-CXCL4).

Chemokine-receptor activation follows a two-site binding mechanism involving interactions of the chemokine $\mathrm{N}$-loop/core with the receptor $\mathrm{N}$ terminus (site $\mathrm{I}$ ) and of the chemokine $\mathrm{N}$ terminus with extracellular/transmembrane residues (site II). The modeled structure for binding of the atypical CC chemokine vMIP-II to CXCR4 appears to support findings that $\mathrm{CC}$ chemokine homodimers cannot bind or activate receptors because the dimerization interface largely coincides with an intermediate recognition site (35-37); however, this may not extend to all chemokine-receptor interactions because N-terminal and core structures in a CC-type heterodimer, for example, OPRAH, may be differently presented to receptors. Instead, binding may involve receptor complexes enabling synergy. Concomitant peptidebased disruption of CCL5-CCL17 and CCR4-CCR5 formation/ function supported the concept that CC-type heterodimers can 


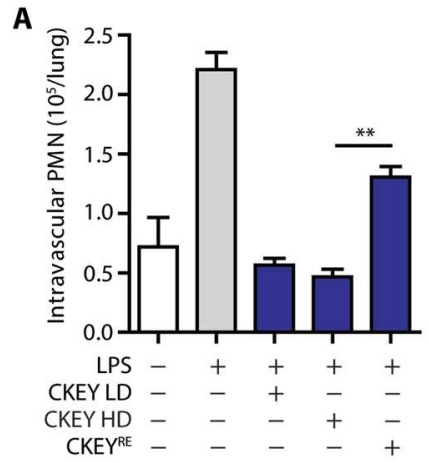

D

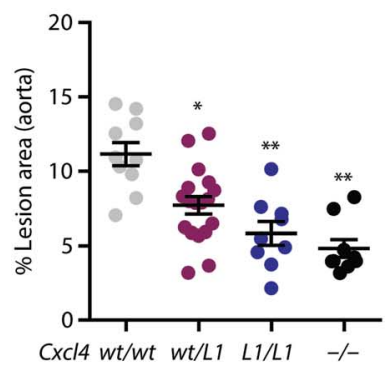

$\mathbf{G}$

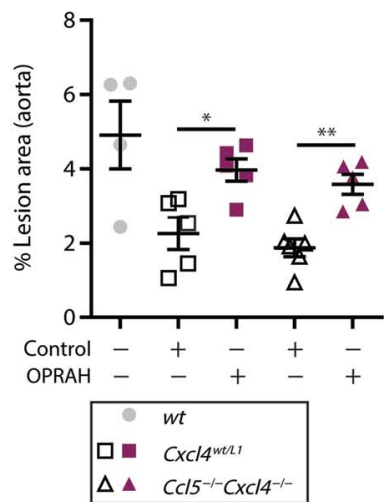

B

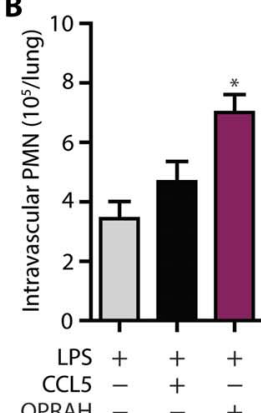

E

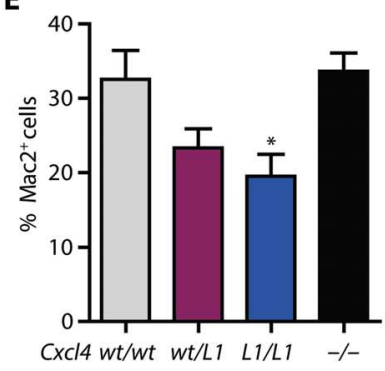

H

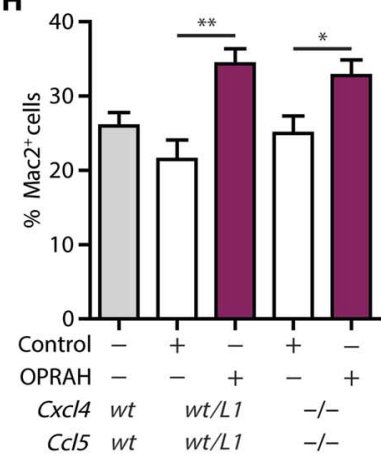

C

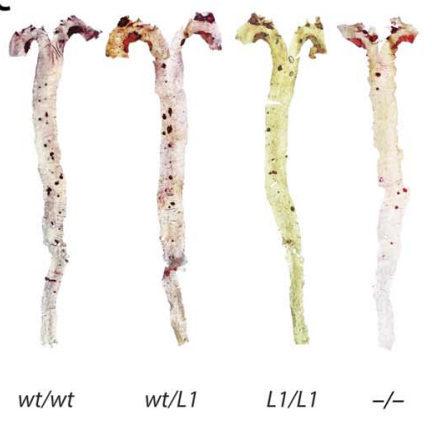

F

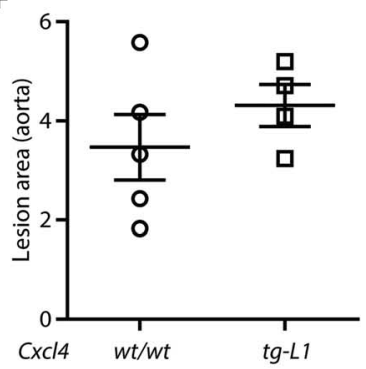

prompt the formation of corresponding receptor complexes, requiring CCR5 transmembrane regions. Furthermore, because chemokine $\mathrm{N}$-terminal residues play an important role in receptor activation, they may also affect the mechanisms of action for heterodimers. For instance, $\mathrm{N}$-terminal sequence variations may cause differences in N-terminal orientation and/or accessibility between CCL17-CCL5 heterodimers and CCL5 homodimers to affect receptor binding and activation. As evidenced from a disulfide-trapped CCL4 variant, dissociation of CCL4 homodimers may be required to accomplish receptor binding and activation by a monomer (37). This may also hold true for chemokine heterodimers, namely, CCL5-CCL17, when addressing respective heterodimers.

The conserved GP motif in CXCL8 can couple sites I and II, dictating both substrates and cross-talk between sites to control receptor activity (38). Conformational coupling was corroborated by a CC mutant of CXCL8 that retained binding via N-loop-site I interactions for CXCR1 activation (39). Moreover, a disulfide-trapped CXCL8 dimer had reduced affinity for CXCR1 due to perturbed binding at Nloop residues, giving rise to a model where binding of a CXC-type dimer triggers conformational changes, leading to release and high-affinity 


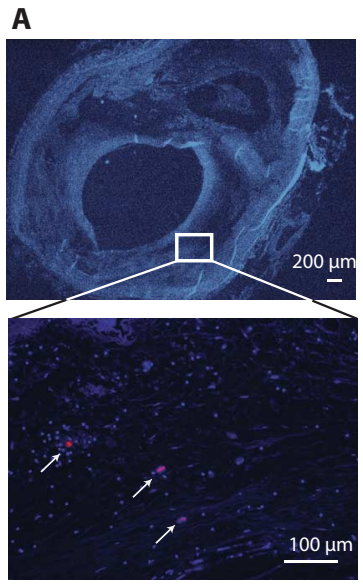

G
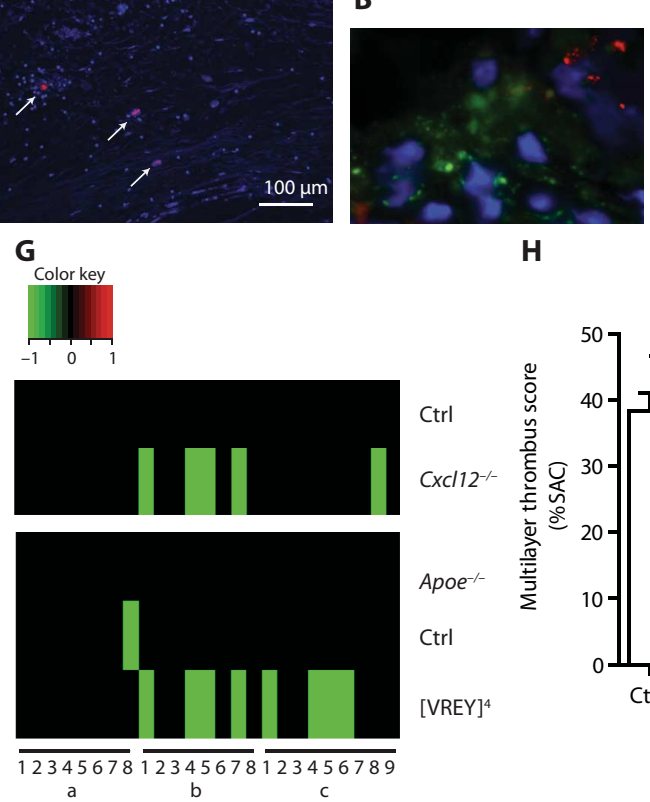

H

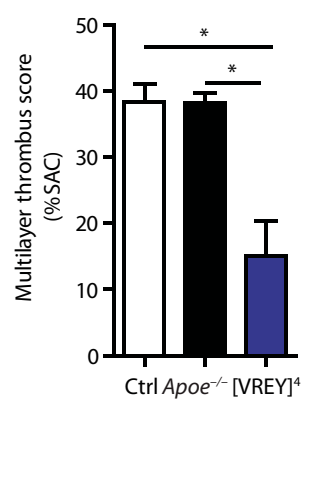

C

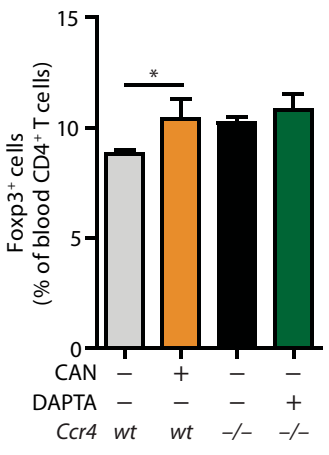

I
D

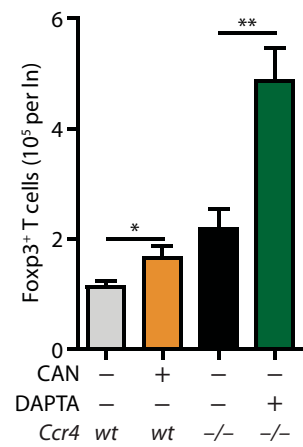

E

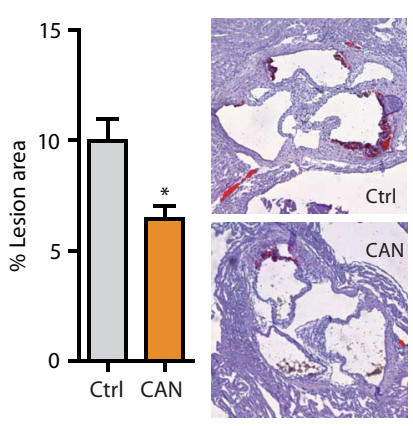

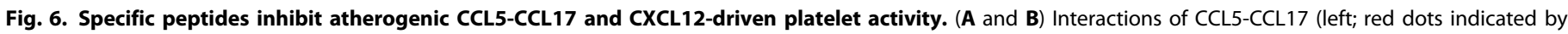

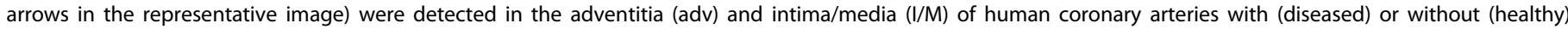

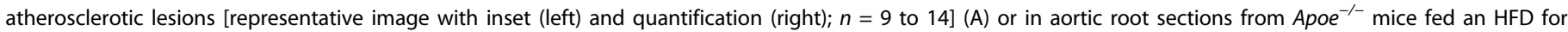

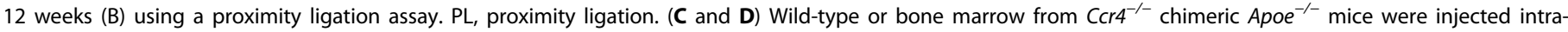

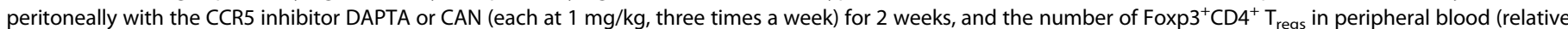

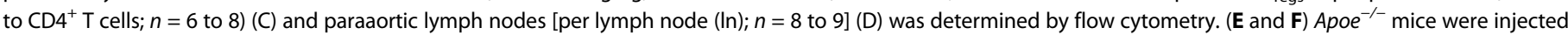

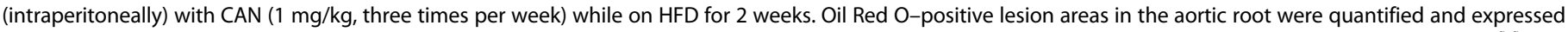

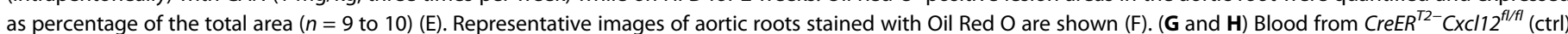

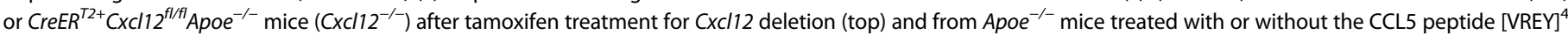

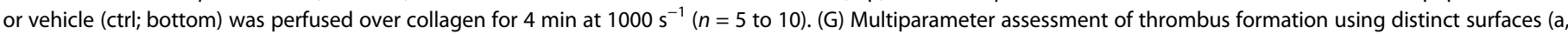

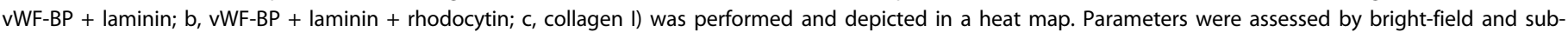

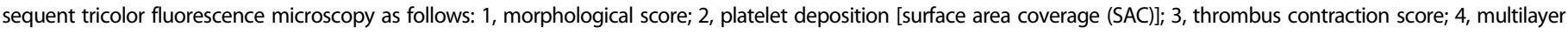

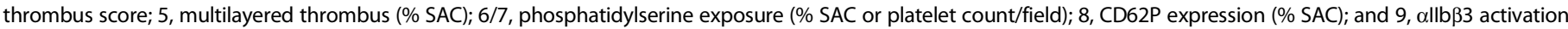

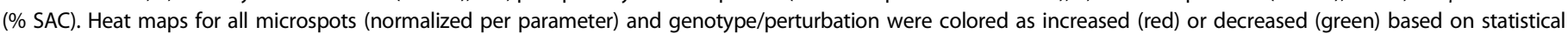

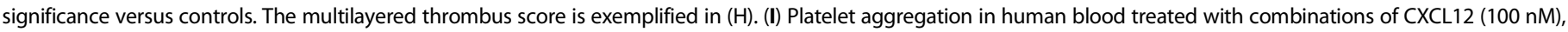

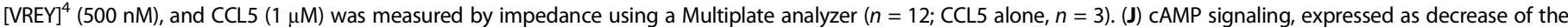

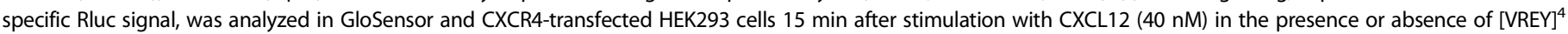

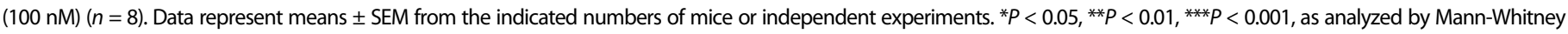
test (A to $C)$, unpaired $t$ test $(D, E$, and $J)$, or Kruskal-Wallis test ( $H$ and $I)$.

receptor binding of a monomer (40). Whether this applies to CC-type heterodimers is unclear; however, findings with OPRAH indicate that dissociation may not be essential for function. Conversely, some receptors, for example, CXCR2, are permissive for dimer binding (41). Macrophage migration inhibitory factor (resembling a CXCL8 dimer) features sites I and II binding to CXCR2 without typical $\mathrm{N}$ termini $(42,43)$. Thus, $\mathrm{N}$ termini may not need to fit fully into the site II pocket.

The increased efficacy of CCL5-bearing heterodimers may be explained by several aspects. CCR5 antagonists inhibited both CCL5-
CXCL4- and CCL5-CCL17-induced arrest. Thus, CC-type heterodimers can modulate CCR5 functionality, converting it into an arrest receptor such as CCR1 (requiring CCL5 oligomers and extracellular loop 3 for sensing) (44-46). As revealed by GAG binding-impaired CXCL4, synergy with CCL5 relies on elevated cell surface presentation, impeding CCR1 internalization to sustain its signaling activity. Formation of chemokine homo- and heterodimers can be affected by GAGs, which may foster or prevent an association of chemokines (6), implying that there is not a single entity of chemokine-chemokine 
interactions but rather that the interactome may vary depending on the presence of soluble or membrane-bound GAGs. Binding of CXCL4 to surface-bound heparin allowed for an additive, likely heteromeric, association of CCL5. The structural basis for CCL5 oligomerization and GAG binding has been elaborated (47). Polymerization creates distinctive grooves that increase GAG avidity; besides the BBXB motif, CCL5 uses another positively charged motif, KKWVR, which supports CCR5 function (44). Models further predict that CXCL4 can assemble heterooligomers with CCL5 featuring an altered surface charge distribution that facilitates GAG binding (47).

Regarding the inhibitory effects of CXC-type heterodimers, it is remarkable that a constitutively homodimeric CXCL12 variant bridged by N-terminal CXCR4 peptides induces calcium signals but inhibits chemotaxis, whereas a monomeric variant remains chemotactic, indicating that dimerization limits monomer-induced chemotaxis (48). Related mechanisms may apply to CXC-type heterodimers, whereby functional activity is impeded by more stable $\beta$-sheet interactions attenuating monomer release. Alternatively, CXC-type heterodimeric interactions may cause conformational alterations, for example, by aligning the $\alpha$-helices (12). Consistent with spectral changes, $\alpha$-helical peptide $[\mathrm{VREY}]^{4}$ inhibited CXCL12 function, mimicking the effects of full-length CCL5.

When assessing the stoichiometry of chemokines, it is reasonable to also consider the role of receptor stoichiometry. Although we detected chemokine receptor heteromers (CCR4-CCR5), the stoichiometry of this association currently remains unknown. Chemokine receptors can cluster in arrays, thereby influencing their surface expression, ligand levels, and function (49). The functional effects observed herein are mediated by distinct mechanisms, depending on which chemokine heterodimer is actually formed, and can involve one agonist receptor or receptor heterodimers. Similar principles may apply to the heterodimerization of chemokines, as for their homooligomerization. In particular, inhibition by CXC-type chemokine heterodimers may favor a 2:1 receptor/chemokine model as a feasible explanation for negative binding cooperativity and transinhibition between chemokine receptor heterodimers (50).

We should point out that the models proposed herein, for instance, with regard to underlying mechanisms by which chemokine heterodimers address their respective receptors, are elaborated to best explain our data but still await direct structural evidence. In addition, we could functionally test and validate only a selected number of chemokine heterodimers but not all possible combinations. Hence, we cannot exclude that some heterodimer pairs may not comply with the functional dichotomy observed for CC- and CXC-type heterodimers.

Several lines of evidence support a functional relevance of heteromer formation in disease models. First, obligate CC-type heterodimers show high efficacy and potency in recruitment assays, namely, OPRAHexacerbated LPS-induced acute lung injury and chronic macrophageladen atherosclerosis. Second, knocking in of CXCL4L1 (not interacting with CCL5) was similarly effective in protecting from atherosclerosis as CXCL4 deficiency, indicating that heterodimer formation with CCL5 was essential for atherogenic activity of CXCL4. Finally, the inhibition

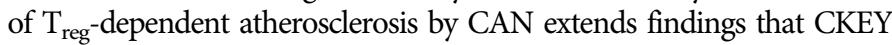
limits atherosclerosis and acute lung injury $(7,11)$ by interfering with residues crucial for heterodimerization.

Notably, structural information regarding the type of interaction and precise location of the interface can be exploited to design peptide inhibitors selectively blocking CC-type heterodimers (as exemplified by CKEY targeting CCL5-CXCL4 and CAN targeting CCL5-CCL17). By contrast, $[\mathrm{VREY}]^{4}$ mimics the inhibitory effects of CXC-type interactions between CCL5 and CXCL12 and attenuates CXCL12-mediated platelet aggregation. Therefore, such CXCL12-targeting peptides could serve as alternative antiplatelet therapeutics. Our paradigms illustrate strategies for specific targeting of heterodimer-mediated functions in the chemokine interactome and may allow for multivalent interference with a diversity of responses in disease-related chemokine microenvironments (fig. S25). The TASP scaffolding used for [VREY $]^{4}$ may also be applicable to bundle different peptides in a polyantagonist approach. Alternatively, sequence hybridization could be used to generate monomolecular polyantagonists, as described for peptide triagonists (51). Our data enable the development of peptide therapeutics based on endogenous sequences that are selective in modulating heteromeric but not primary receptor interactions of chemokines and could thus lack many adverse effects associated with direct receptor-ligand antagonism.

\section{MATERIALS AND METHODS Study design}

The overall objective of this study was to establish a comprehensive map of the chemokine interactome and to identify the structurefunction relationship of different chemokine heterodimer types. Subsequent objectives were the design of specific peptides to disrupt functional synergy or to mimic the inhibitory effects of heterodimers and their validation in models of inflammation and atherosclerosis. For mouse studies, a power analysis was performed using BiAS software (version 11.02). We assumed a detectable biological difference of at least $50 \%$ among up to four groups with an SD of $<15 \%$, an $\alpha$ of 0.05 , and a resultant power of 0.8 . On the basis of these assumptions, at least four mice were included in each group. The number of biological replicates for each data point is included in the figure legends. All data were included (no outliers were excluded).

\section{Ligand blots}

Chemokines $(1 \mu \mathrm{g})$ were spotted on a nitrocellulose membrane, which was immersed overnight with the soluble chemokine ( 1 to $5 \mu \mathrm{g} / \mathrm{ml}$ ), and reacted with biotinylated antibodies and streptavidin-conjugated horseradish peroxidase and enhanced chemiluminescence substrate (Thermo Fisher Scientific) essentially as described (52). The chemiluminescence signal was digitally recorded and analyzed by the luminescence image analyzer LAS-3000 and Multi Gauge software (Fuji Photo Film).

\section{Surface plasmon resonance}

SPR was performed using a Biacore X100 instrument (GE Healthcare Europe $\mathrm{GmbH}$ ) and neutravidin-modified $\mathrm{C} 1$ sensor chips (52). Biotinylated chemokines were immobilized on flow cells to $0.3 \times 10^{3}$ to $1.3 \times 10^{3} \mathrm{RU}$. Screening of chemokine binding was performed at $100 \mathrm{nM}$ in HBS-EP+ buffer at $90 \mu \mathrm{l} / \mathrm{min}$ after $20 \mathrm{~s}$. Apparent affinities were calculated from on and off rates after fitting of the curves obtained from increasing analyte concentrations using a 1:1 Langmuir interaction model (BIAevaluation software). To assess the inhibitory capacity of peptides, we recorded the binding of chemokines $(20 \mathrm{nM})$ incubated with increasing peptide concentrations and calculated the percentage inhibition. Halfmaximal inhibitory concentrations $\left(\mathrm{IC}_{50}\right)$ were determined by four-parameter logistic nonlinear regression (GraphPad version 5.0).

\section{NMR spectroscopy}

NMR samples were prepared in Wilmad 3-mm NMR tubes (160 $\mu \mathrm{l})$. Typically, chemokine samples were buffer-exchanged and concentrated 
into $25 \mathrm{mM}$ sodium acetate- $\mathrm{d}_{3}(\mathrm{pH} 4.5)$ containing $0.1 \mathrm{mM}$ EDTA and $0.2 \mathrm{mM}$ sodium azide, through five ultracentrifugation steps over Amicon Ultra-4 3-kDa filter devices (Merck Millipore). Concentrations of final stock solutions were determined using a NanoDrop 2000 spectrophotometer (Thermo Fisher Scientific). Mixtures of chemokines at defined molar ratios were prepared from these stock solutions, and $5 \%(\mathrm{v} / \mathrm{v}) \mathrm{D}_{2} \mathrm{O}$ was added for field locking, together with a trace of DSS (4,4-dimethyl-4-silapentane-1-sulfonic acid) as internal chemical shift standard. NMR spectra $\left[{ }^{1} \mathrm{H}^{15} \mathrm{~N}\right.$ HSQC with flip-back pulse, DIPSI (decoupling in the presence of scalar interactions), and NOESY (nuclear Overhauser effect spectroscopy)] were recorded at $37^{\circ} \mathrm{C}$ on Bruker Avance III HD 700- and 850-MHz spectrometers equipped with cryogenically cooled TCI probes. Spectra were processed and analyzed using Bruker TopSpin 3.2 and Sparky 3.114 software (T. D. Goddard, D. G. Kneller, SPARKY 3, and the University of California, San Francisco).

Resonance assignments of CCL $5^{\mathrm{E} 66 \mathrm{~S}}$ and CXCL12 were performed by two-dimensional (2D) NOESY and 3D-edited NOESY spectra. Because $\mathrm{CCL} 5^{\mathrm{E} 66 \mathrm{~S}}$ show homodimers and monomers that are in slow exchange on the NMR chemical shift time scale, resonances for both dimers and monomers are observed and well resolved in HSQC spectra, allowing assessment of effects on both states (22). Chemical shift differences $(\Delta \delta)$ induced upon binding were calculated as follows: $\left[\left(\Delta^{1} \mathrm{H}\right)^{2}\right]^{1 / 2}+\left[\left(0.25 \Delta^{15} \mathrm{~N}\right)^{2}\right]^{1 / 2}$ (in ${ }^{1} \mathrm{H}$ ppm). $\Delta$ Intensity was calculated as follows: $1-\mathrm{Int}_{\mathrm{i}} / \mathrm{Int}_{\mathrm{o}}$, where $\mathrm{Int}_{\mathrm{i}}$ is the resonance intensity of CCL $5^{\mathrm{E} 66 \mathrm{~S}}$ or CXCL12 resonances in the presence of the other chemokine, and $\mathrm{Int}_{\mathrm{o}}$ is the intensity of CCL5 $5^{\mathrm{E} 6 \mathrm{SS}}$ or CXCL12 resonances in its absence.

\section{Statistical analysis}

Data are expressed as means \pm SEM unless otherwise specified. If D'Agostino-Pearson omnibus and/or Shapiro-Wilk normality test indicated Gaussian distribution, an unpaired $t$ test for side-by-side comparisons or one-way ANOVA with Newman-Keuls posttest for multiple comparisons was performed. Otherwise, Mann-Whitney tests for side-by-side comparisons and Kruskal-Wallis test with Dunn's posttest for multiple comparisons were performed using GraphPad Prism version 5.04 for Windows (GraphPad Software).

For all other Materials and Methods, please see Supplementary Materials.

\section{SUPPLEMENTARY MATERIALS}

www.sciencetranslationalmedicine.org/cgi/content/full/9/384/eaah6650/DC1 Materials and Methods

Fig. S1. Representative ligand blot and densitometric values.

Fig. S2. Interaction of CCL5 and CCL5 $5^{\mathrm{E} 66 \mathrm{~S}}$ with CCL17 analyzed by SPR.

Fig. S3. CXCL4 ${ }^{\mathrm{K50E}}$ - and $\mathrm{CCL} 17$-induced changes in the HSQC spectrum of $\mathrm{CCL}^{\mathrm{E} 66 \mathrm{~S}}$

Fig. S4. Chemical shift and resonance intensity changes of $\mathrm{CCL} 5^{\mathrm{E} 665}$ residues.

Fig. S5. CXCL4 ${ }^{\mathrm{K} 50 \mathrm{E}}$-induced changes in the HSQC spectrum of CCL5.

Fig. S6. Concentration-dependent formation of CC- versus CXC-type heterodimers.

Fig. S7. CCL5-induced changes in HSQC spectra of CXCL12.

Fig. S8. Structural models of CC-type and CXC-type heterodimers.

Fig. S9. Targeting of CCL5-CXCL4 heterodimer by CKEY.

Fig. S10. Interaction of CAN with CCL17.

Fig. S11. Targeting of CCL5-CCL17 heterodimer by CAN.

Fig. S12. Efficient targeting of CCL5-CXCL12 heterodimer by [VREY] ${ }^{4}$.

Fig. S13. Dose dependency of T cell chemotaxis elicited by single chemokines.

Fig. S14. Differential effects of chemokine combinations on T cell chemotaxis.

Fig. S15. Differential effects of chemokine heterodimerization on leukocyte recruitment.

Fig. S16. Microscopy of chemokine interactions at the cell surface.

Fig. S17. Mechanisms for synergy of chemokine heterodimers.

Fig. S18. Effect of heterodimers on signaling pathways, receptor affinity, and internalization. Fig. S19. CCL5-CXCL4 heterodimers bind to heparin and prevent arrestin recruitment.
Fig. S20. Relevance of chemokine heterodimer formation in acute lung injury. Fig. S21. Generation of mice deficient in $\mathrm{CxCl} 4$ or $\mathrm{CxCl12}$ or carrying $\mathrm{CxCl} / 4$ variants. Fig. S22. Analysis of atherosclerotic lesion in mice expressing $\mathrm{Cxcl} 4$ or its variant. Fig. S23. Composition of aortic root lesions in mice expressing Cxcl4 or its variant. Fig. S24. Obligate chemokine heterodimers mimic natural heterodimers. Fig. S25. Summary scheme.

Table S1. Densitometry analysis of bidirectional immunoligand blotting. Table S2. Kinetic analysis of chemokine binding by SPR.

Table S3. Weighted averaging of $\Delta$ intensity changes and MDS-based in silico modeling. Table S4. Sequence and origin of peptide inhibitors.

Table S5. CCL5-derived peptides specifically inhibit heterodimer formation. Table S6. Lipid parameters and peripheral blood counts of different CXCL4 genotypes. Table S7. Effect of OPRAH on lipid parameters and peripheral blood counts. References (53-63)

\section{REFERENCES AND NOTES}

1. A. Zlotnik, O. Yoshie, The chemokine superfamily revisited. Immunity 36, 705-716 (2012).

2. F. Bachelerie, A. Ben-Baruch, A. M. Burkhardt, C. Combadiere, J. M. Farber, G. J. Graham, R. Horuk, A. H. Sparre-Ulrich, M. Locati, A. D. Luster, A. Mantovani, K. Matsushima, P. M. Murphy, R. Nibbs, H. Nomiyama, C. A. Power, A. E. I. Proudfoot, M. M. Rosenkilde, A. Rot, S. Sozzani, M. Thelen, O. Yoshie, A. Zlotnik, International Union of Basic and Clinical Pharmacology. LXXXIX. Update on the extended family of chemokine receptors and introducing a new nomenclature for atypical chemokine receptors. Pharmacol. Rev. 66, 1-79 (2014).

3. C. Weber, H. Noels, Atherosclerosis: Current pathogenesis and therapeutic options. Nat. Med. 17, 1410-1422 (2011).

4. R. R. Koenen, C. Weber, Therapeutic targeting of chemokine interactions in atherosclerosis. Nat. Rev. Drug Discov. 9, 141-153 (2010).

5. E. J. Fernandez, E. Lolis, Structure, function, and inhibition of chemokines. Annu. Rev. Pharmacol. Toxicol. 42, 469-499 (2002).

6. S. E. Crown, Y. Yu, M. D. Sweeney, J. A. Leary, T. M. Handel, Heterodimerization of CCR2 chemokines and regulation by glycosaminoglycan binding. J. Biol. Chem. 281, 25438-25446 (2006).

7. R. R. Koenen, P. von Hundelshausen, I. V. Nesmelova, A. Zernecke, E. A. Liehn, A. Sarabi, B. K. Kramp, A. M. Piccinini, S. R. Paludan, M. A. Kowalska, A. J. Kungl, T. M. Hackeng, K. H. Mayo, C. Weber, Disrupting functional interactions between platelet chemokines inhibits atherosclerosis in hyperlipidemic mice. Nat. Med. 15, 97-103 (2009).

8. I. V. Nesmelova, Y. Sham, J. Gao, K. H. Mayo, CXC and CC chemokines form mixed heterodimers: Association free energies from molecular dynamics simulations and experimental correlations. J. Biol. Chem. 283, 24155-24166 (2008).

9. A. Z. Dudek, I. Nesmelova, K. Mayo, C. M. Verfaillie, S. Pitchford, A. Slungaard, Platelet factor 4 promotes adhesion of hematopoietic progenitor cells and binds IL-8: Novel mechanisms for modulation of hematopoiesis. Blood 101, 4687-4694 (2003).

10. I. V. Nesmelova, Y. Sham, A. Z. Dudek, L. I. van Eijk, G. Wu, A. Slungaard, F. Mortari, A. W. Griffioen, K. H. Mayo, Platelet factor 4 and interleukin-8 CXC chemokine heterodimer formation modulates function at the quaternary structural level. J. Biol. Chem. 280, 4948-4958 (2005).

11. J. Grommes, J. E. Alard, M. Drechsler, S. Wantha, M. Morgelin, W. M. Kuebler, M. Jacobs, P. von Hundelshausen, P. Markart, M. Wygrecka, K. T. Preissner, T. M. Hackeng, R. R. Koenen, C. Weber, O. Soehnlein, Disruption of platelet-derived chemokine heteromers prevents neutrophil extravasation in acute lung injury. Am. J. Respir. Crit. Care Med. 185, 628-636 (2012).

12. J. Carlson, S. A. Baxter, D. Dréau, I. V. Nesmelova, The heterodimerization of plateletderived chemokines. Biochim. Biophys. Acta 1834, 158-168 (2013).

13. S. Sebastiani, G. Danelon, B. Gerber, M. Uguccioni, CCL22-induced responses are powerfully enhanced by synergy inducing chemokines via CCR4: Evidence for the involvement of first $\beta$-strand of chemokine. Eur. J. Immunol. 35, 746-756 (2005).

14. K. Kuscher, G. Danelon, S. Paoletti, L. Stefano, M. Schiraldi, V. Petkovic, M. Locati, B. O. Gerber, M. Uguccioni, Synergy-inducing chemokines enhance CCR2 ligand activities on monocytes. Eur. J. Immunol. 39, 1118-1128 (2009).

15. M. Ren, Q. Guo, L. Guo, M. Lenz, F. Qian, R. R. Koenen, H. Xu, A. B. Schilling, C. Weber, R. D. Ye, A. R. Dinner, W.-J. Tang, Polymerization of MIP-1 chemokine (CCL3 and CCL4) and clearance of MIP-1 by insulin-degrading enzyme. EMBO J. 29, 3952-3966 (2010).

16. W. G. Liang, M. Ren, F. Zhao, W.-J. Tang, Structures of human CCL18, CCL3, and CCL4 reveal molecular determinants for quaternary structures and sensitivity to insulindegrading enzyme. J. Mol. Biol. 427, 1345-1358 (2015).

17. A. Zernecke, C. Weber, Chemokines in atherosclerosis: Proceedings resumed. Arterioscler. Thromb. Vasc. Biol. 34, 742-750 (2014).

18. S. Struyf, M. D. Burdick, P. Proost, J. Van Damme, R. M. Strieter, Platelets release CXCL4L1, a nonallelic variant of the chemokine platelet factor-4/CXCL4 and potent inhibitor of angiogenesis. Circ. Res. 95, 855-857 (2004). 
19. J.-H. Kuo, Y. P. Chen, J.-S. Liu, A. Dubrac, C. Quemener, H. Prats, A. Bikfalvi, W.-g. Wu, S.-C. Sue, Alternative C-terminal helix orientation alters chemokine function: Structure of the anti-angiogenic chemokine, CXCL4L1. J. Biol. Chem. 288, 13522-13533 (2013).

20. K. H. Mayo, E. Ilyina, V. Roongta, M. Dundas, J. Joseph, C. K. Lai, T. Maione, T. J. Daly, Heparin binding to platelet factor-4. An NMR and site-directed mutagenesis study: Arginine residues are crucial for binding. Biochem. J. 312 (Pt. 2), 357-365 (1995).

21. L. G. Czaplewski, J. McKeating, C. J. Craven, L. D. Higgins, V. Appay, A. Brown, T. Dudgeon, L. A. Howard, T. Meyers, J. Owen, S. R. Palan, P. Tan, G. Wilson, N. R. Woods, C. M. Heyworth, B. I. Lord, D. Brotherton, R. Christison, S. Craig, S. Cribbes, R. M. Edwards, S. J. Evans, R. Gilbert, P. Morgan, E. Randle, N. Schofield, P. G. Varley, J. Fisher, J. P. Waltho, M. G. Hunter, Identification of amino acid residues critical for aggregation of human CC chemokines macrophage inflammatory protein (MIP)- $1 \alpha$, MIP-1 $\beta$, and RANTES. Characterization of active disaggregated chemokine variants. J. Biol. Chem. 274, 16077-16084 (1999).

22. T. M. Handel, P. J. Domaille, Heteronuclear $\left({ }^{1} \mathrm{H},{ }^{13} \mathrm{C},{ }^{15} \mathrm{~N}\right) \mathrm{NMR}$ assignments and solution structure of the monocyte chemoattractant protein-1 (MCP-1) dimer. Biochemistry 35, 6569-6584 (1996).

23. K. H. Mayo, M. J. Chen, Human platelet factor 4 monomer-dimer-tetramer equilibria investigated by $1 \mathrm{H}$ NMR spectroscopy. Biochemistry 28, 9469-9478 (1989).

24. A. D. Luster, S. M. Greenberg, P. Leder, The IP-10 chemokine binds to a specific cell surface heparan sulfate site shared with platelet factor 4 and inhibits endothelial cell proliferation. J. Exp. Med. 182, 219-231 (1995).

25. P. E. Dawson, S. B. H. Kent, Convenient total synthesis of a 4-helix template-assembled synthetic protein (TASP) molecule by chemoselective ligation. J. Am. Chem. Soc. 115, 7263-7266 (1993).

26. O. Söderberg, M. Gullberg, M. Jarvius, K. Ridderstråle, K.-J. Leuchowius, J. Jarvius, K. Wester, P. Hydbring, F. Bahram, L.-G. Larsson, U. Landegren, Direct observation of individual endogenous protein complexes in situ by proximity ligation. Nat. Methods $\mathbf{3}$, 995-1000 (2006).

27. P. Hernanz-Falcón, J. M. Rodríguez-Frade, A. Serrano, D. Juan, A. del Sol, S. F. Soriano, F. Roncal, L. Gomez, A. Valencia, A. C. Martínez-A, M. Mellado, Identification of amino acid residues crucial for chemokine receptor dimerization. Nat. Immunol. 5, 216-223 (2004).

28. M. Mellado, J. M. Rodríguez-Frade, A. J. Vila-Coro, S. Fernández, A. Martín de Ana, D. R. Jones, J. L. Torán, A. C. Martínez-A, Chemokine receptor homo- or heterodimerization activates distinct signaling pathways. EMBO J. 20, 2497-2507 (2001).

29. G. E. Callander, W. G. Thomas, R. A. Bathgate, Prolonged RXFP1 and RXFP2 signaling can be explained by poor internalization and a lack of $\beta$-arrestin recruitment. Am. J. Physiol. Cell Physiol. 296, C1058-C1066 (2009).

30. C. Weber, S. Meiler, Y. Döring, M. Koch, M. Drechsler, R. T. A. Megens, Z. Rowinska, K. Bidzhekov, C. Fecher, E. Ribechini, M. A. M. J. van Zandvoort, C. J. Binder, I. Jelinek, M. Hristov, L. Boon, S. Jung, T. Korn, M. B. Lutz, I. Forster, M. Zenke, T. Hieronymus, T. Junt, A. Zernecke, CCL17-expressing dendritic cells drive atherosclerosis by restraining regulatory T cell homeostasis in mice. J. Clin. Invest. 121, 2898-2910 (2011).

31. D. F. J. Ketelhuth, G. K. Hansson, Adaptive response of $T$ and $B$ cells in atherosclerosis. Circ. Res. 118, 668-678 (2016).

32. M. Vidal, How much of the human protein interactome remains to be mapped? Sci. Signal. 9, eg7 (2016).

33. K. M. Ansel, V. N. Ngo, P. L. Hyman, S. A. Luther, R. Forster, J. D. Sedgwick, J. L. Browning, M. Lipp, J. G. Cyster, A chemokine-driven positive feedback loop organizes lymphoid follicles. Nature 406, 309-314 (2000).

34. K. M. Ansel, R. B. S. Harris, J. G. Cyster, CXCL13 is required for B1 cell homing, natural antibody production, and body cavity immunity. Immunity 16, 67-76 (2002).

35. L. Qin, I. Kufareva, L. G. Holden, C. Wang, Y. Zheng, C. Zhao, G. Fenalti, H. Wu, G. W. Han, V. Cherezov, R. Abagyan, R. C. Stevens, T. M. Handel, Crystal structure of the chemokine receptor CXCR4 in complex with a viral chemokine. Science 347, 1117-1122 (2015).

36. L. J. Drury, J. J. Ziarek, S. Gravel, C. T. Veldkamp, T. Takekoshi, S. T. Hwang, N. Heveker, B. F. Volkman, M. B. Dwinell, Monomeric and dimeric CXCL12 inhibit metastasis through distinct CXCR4 interactions and signaling pathways. Proc. Natl. Acad. Sci. U.S.A. 108, 17655-17660 (2011).

37. H. Jin, X. Shen, B. R. Baggett, X. Kong, P. J. LiWang, The human CC chemokine MIP-1 $\beta$ dimer is not competent to bind to the CCR5 receptor. J. Biol. Chem. 282, 27976-27983 (2007).

38. P. R. B. Joseph, K. V. Sawant, A. Isley, M. Pedroza, R. P. Garofalo, R. M. Richardson, K. Rajarathnam, Dynamic conformational switching in the chemokine ligand is essential for G-protein-coupled receptor activation. Biochem. J. 456, 241-251 (2013).

39. K. Rajarathnam, G. N. Prado, H. Fernando, I. Clark-Lewis, J. Navarro, Probing receptor binding activity of interleukin-8 dimer using a disulfide trap. Biochemistry $45,7882-7888$ (2006).

40. A. Ravindran, P. R. B. Joseph, K. Rajarathnam, Structural basis for differential binding of the interleukin- 8 monomer and dimer to the CXCR1 N-domain: Role of coupled interactions and dynamics. Biochemistry 48, 8795-8805 (2009).
41. A. Ravindran, K. V. Sawant, J. Sarmiento, J. Navarro, K. Rajarathnam, Chemokine CXCL1 dimer is a potent agonist for the CXCR2 receptor. J. Biol. Chem. 288, 12244-12252 (2013).

42. S. Kraemer, H. Lue, A. Zernecke, A. Kapurniotu, E. Andreetto, R. Frank, B. Lennartz, C. Weber, J. Bernhagen, MIF-chemokine receptor interactions in atherogenesis are dependent on an N-loop-based 2-site binding mechanism. FASEB J. 25, 894-906 (2011).

43. L. Xu, Y. Li, D. Li, P. Xu, S. Tian, H. Sun, H. Liu, T. Hou, Exploring the binding mechanisms of MIF to CXCR2 using theoretical approaches. Phys. Chem. Chem. Phys. 17, 3370-3382 (2015).

44. B. K. Kramp, R. T. Megens, A. Sarabi, S. Winkler, D. Projahn, C. Weber, R. R. Koenen, $P$. von Hundelshausen, Exchange of extracellular domains of CCR1 and CCR5 reveals confined functions in CCL5-mediated cell recruitment. Thromb. Haemost. 110, 795-806 (2013).

45. C. Weber, K. S. Weber, C. Klier, S. Gu, R. Wank, R. Horuk, P. J. Nelson, Specialized roles of the chemokine receptors CCR1 and CCR5 in the recruitment of monocytes and $\mathrm{T}_{\mathrm{H}} 1$-like/ CD45RO ${ }^{+}$T cells. Blood 97, 1144-1146 (2001).

46. T. Baltus, K. S. Weber, Z. Johnson, A. E. I. Proudfoot, C. Weber, Oligomerization of RANTES is required for CCR1-mediated arrest but not CCR5-mediated transmigration of leukocytes on inflamed endothelium. Blood 102, 1985-1988 (2003).

47. W. G. Liang, C. G. Triandafillou, T. Y. Huang, M. M. L. Zulueta, S. Banerjee, A. R. Dinner, S. C. Hung, W.-J. Tang, Structural basis for oligomerization and glycosaminoglycan binding of CCL5 and CCL3. Proc. Natl. Acad. Sci. U.S.A. 113, 5000-5005 (2016).

48. C. T. Veldkamp, C. Seibert, F. C. Peterson, N. B. De la Cruz, J. C. Haugner III, H. Basnet, T. P. Sakmar, B. F. Volkman, Structural basis of CXCR4 sulfotyrosine recognition by the chemokine SDF-1/CXCL12. Sci. Signal. 1, ra4 (2008).

49. M. Thelen, L. M. Munoz, J. M. Rodríguez-Frade, M. Mellado, Chemokine receptor oligomerization: Functional considerations. Curr. Opin. Pharmacol. 10, 38-43 (2010).

50. P. Cutolo, N. Basdevant, G. Bernadat, F. Bachelerie, T. Ha-Duong, Interaction of chemokine receptor CXCR4 in monomeric and dimeric state with its endogenous ligand CXCL12: Coarse-grained simulations identify differences. J. Biomol. Struct. Dyn. 35, 399-412 (2017).

51. B. Finan, B. Yang, N. Ottaway, D. L. Smiley, T. Ma, C. Clemmensen, J. Chabenne, L. Zhang K. M. Habegger, K. Fischer, J. E. Campbell, D. Sandoval, R. J. Seeley, K. Bleicher, S. Uhles, W. Riboulet, J. Funk, C. Hertel, S. Belli, E. Sebokova, K. Conde-Knape, A. Konkar, D. J. Drucker, V. Gelfanov, P. T. Pfluger, T. D. Muller, D. Perez-Tilve, R. D. DiMarchi, M. H. Tschöp, A rationally designed monomeric peptide triagonist corrects obesity and diabetes in rodents. Nat. Med. 21, 27-36 (2015)

52. P. von Hundelshausen, R. R. Koenen, M. Sack, S. F. Mause, W. Adriaens, A. E. I. Proudfoot T. M. Hackeng, C. Weber, Heterophilic interactions of platelet factor 4 and RANTES promote monocyte arrest on endothelium. Blood 105, 924-930 (2005).

53. J. Feierler, M. Wirth, B. Welte, S. Schussler, M. Jochum, A. Faussner, Helix 8 plays a crucial role in bradykinin $B_{2}$ receptor trafficking and signaling. J. Biol. Chem. 286, 43282-43293 (2011).

54. A. E. I. Proudfoot, F. Borlat, Purification of recombinant chemokines from E. coli. Methods Mol. Biol. 138, 75-87 (2000).

55. A. E. I. Proudfoot, T. M. Handel, Z. Johnson, E. K. Lau, P. LiWang, I. Clark-Lewis, F. Borlat T. N. C. Wells, M. H. Kosco-Vilbois, Glycosaminoglycan binding and oligomerization are essential for the in vivo activity of certain chemokines. Proc. Natl. Acad. Sci. U.S.A. 100, 1885-1890 (2003)

56. T. M. Hackeng, J. H. Griffin, P. E. Dawson, Protein synthesis by native chemical ligation: Expanded scope by using straightforward methodology. Proc. Natl. Acad. Sci. U.S.A. 96, 10068-10073 (1999)

57. P. E. Dawson, T. W. Muir, I. Clark-Lewis, S. B. Kent, Synthesis of proteins by native chemical ligation. Science 266, 776-779 (1994).

58. S. M. Agten, D. Suylen, H. Ippel, M. Kokozidou, G. Tans, P. van de Vijver, R. R. Koenen, T. M. Hackeng, Chemoselective oxime reactions in proteins and peptides by using an optimized oxime strategy: The demise of levulinic acid. ChemBioChem 14, 2431-2434 (2013).

59. S. M. Agten, R. R. Koenen, H. Ippel, V. Eckardt, P. von Hundelshausen, K. H. Mayo, C. Weber, T. M. Hackeng, Probing functional heteromeric chemokine protein-protein interactions through conformation-assisted oxime ligation. Angew. Chem. Int. Ed. Engl. 55, 14963-14966 (2016).

60. J.-E. Alard, A. Ortega-Gomez, K. Wichapong, D. Bongiovanni, M. Horckmans, R. T. Megens, G. Leoni, B. Ferraro, J. Rossaint, N. Paulin, J. Ng, H. Ippel, D. Suylen, R. Hinkel, X. Blanchet, F. Gaillard, M. D'Amico, P. von Hundelshausen, A. Zarbock, C. Scheiermann, T. M. Hackeng, S. Steffens, C. Kupatt, G. A. F. Nicolaes, C. Weber, O. Soehnlein, Recruitment of classical monocytes can be inhibited by disturbing heteromers of neutrophil HNP1 and platelet CCL5. Sci. Transl. Med. 7, 317ra196 (2015).

61. K. Wichapong, J.-E. Alard, A. Ortega-Gomez, C. Weber, T. M. Hackeng, O. Soehnlein, G. A. F. Nicolaes, Structure-based design of peptidic inhibitors of the interaction between 
CC chemokine ligand 5 (CCL5) and human neutrophil peptides 1 (HNP1). J. Med. Chem. 59, 4289-4301 (2016).

62. R. Van Kruchten, J. M. E. M. Cosemans, J. W. M. Heemskerk, Measurement of whole blood thrombus formation using parallel-plate flow chambers-A practical guide. Platelets 23, 229-242 (2012).

63. S. M. de Witt, F. Swieringa, R. Cavill, M. M. Lamers, R. van Kruchten, T. Mastenbroek, C. Baaten, S. Coort, N. Pugh, A. Schulz, I. Scharrer, K. Jurk, B. Zieger, K. J. Clemetson, R. W. Farndale, J. W. Heemskerk, J. M. Cosemans, Identification of platelet function defects by multi-parameter assessment of thrombus formation. Nat. Commun. 5, 4257 (2014).

Acknowledgments: We thank A. Proudfoot for providing Met-RANTES and CCL5 mutants and D. Suylen for chemical synthesis of $C X C L 4^{R>Q}$. Funding: This study was supported by Deutsche Forschungsgemeinschaft (DFG) (SFB1123-A1 to C.W. and Y.D., SFB1123-A2 to P.v.H. and R.R.K., SFB1123-A6 to O.S. and M.D., SFB1123-B5 to Y.D. and O.S., SFB1123-Z1 to R.T.A.M., SFB914-B08 to O.S. and C.W., and INST 409/150-1 FUGG to C.W. and R.T.A.M.), the European Research Council (ERC Advanced Grants nos. 249929 and 692511 to C.W.), Nederlandse Organisatie voor Wetenschappelijk Onderzoek (NWO) (VICl project no. 91810606 to C.W. and VIDI projects nos. 91712303 to O.S. and 91712358 to R.R.K.), Fondation Leducq [Transatlantic Network of Excellence CVGeneF(x) to C.W.], and project INCOAG from the Center for Translational Molecular Medicine to J.W.M.H. C.W. holds a van de Laar professorship at the Cardiovascular Research Institute Maastricht, Maastricht, Netherlands. Author contributions: P.v.H. conceived the study, analyzed and interpreted the experiments, and wrote the manuscript. S.M.A. designed and synthesized the peptides and obligate heterodimers. V.E. performed the biochemical and functional experiments. M.M.S. performed the functional and animal experiments. X.B. generated the recombinant proteins and performed SPR studies. H.I. performed the NMR experiments. K.B. generated the transgenic mice. K.W. performed in silico analyses. A.F. performed signaling studies. A.O.-G. analyzed CCR1 expression in mice. R.T.A.M. performed stimulated emission depletion imaging experiments. C.N., M.D., J.P.v.G., and R.N. performed the animal experiments. H.L. designed and generated the proteins. I.D., J.L., G.A.F.N., Y.D., O.S., E.L., J.W.M.H., and R.R.K. analyzed the data. K.H.M. and T.M.H. supervised the study, analyzed the data, and made critical revisions to the manuscript. C.W. conceived and supervised the study and wrote the manuscript. Competing interests: P.v.H., R.R.K., and C.W. are inventors on patents (filed or granted under application no. ES20100181077T) held by RWTH Aachen University that cover CKEY and related peptides and are shareholders of Carolus Therapeutics Inc.

Submitted 2 August 2016

Resubmitted 18 January 2017

Accepted 1 March 2017

Published 5 April 2017

10.1126/scitransImed.aah6650

Citation: P. von Hundelshausen, S. M. Agten, V. Eckardt, X. Blanchet, M. M. Schmitt, H. Ippel, C. Neideck, K. Bidzhekov, J. Leberzammer, K. Wichapong, A. Faussner, M. Drechsler, J. Grommes, J. P. van Geffen, H. Li, A. Ortega-Gomez, R. T. A. Megens, R. Naumann, I. Dijkgraaf, G. A. F. Nicolaes, Y. Döring, O. Soehnlein, E. Lutgens, J. W. M. Heemskerk, R. R. Koenen, K. H. Mayo, T. M. Hackeng, C. Weber, Chemokine interactome mapping enables tailored intervention in acute and chronic inflammation. Sci. Transl. Med. 9 eaah6650 (2017). 


\title{
Science Translational Medicine
}

\section{Chemokine interactome mapping enables tailored intervention in acute and chronic inflammation}

Philipp von HundelshausenStijn M. AgtenVeit EckardtXavier BlanchetMartin M. SchmittHans IppelCarlos NeideckKiril BidzhekovJulian LeberzammerKanin WichapongAlexander FaussnerMaik DrechslerJochen GrommesJohanna P. van GeffenHe LiAlmudena Ortega-GomezRemco T. A. MegensRonald NaumannIngrid DijkgraafGerry A. F. NicolaesYvonne DöringOliver SoehnleinEsther LutgensJohan W. M. HeemskerkRory R. KoenenKevin H. MayoTilman M. HackengChristian Weber

Sci. Transl. Med., 9 (384), eaah6650. • DOI: 10.1126/scitranslmed.aah6650

\begin{abstract}
Hampering heterodimers interrupts inflammation
Inflammation is dependent on the recruitment of cells responding to chemokines. Von Hundelshausen et al. cataloged how human chemokines interact with each other and found that certain kinds of chemokine pairs can activate or inhibit receptor signaling. These chemokine heterodimers were shown to be active in mouse models of acute and chronic inflammation, which were ameliorated by treatment with a peptide designed to disrupt the chemokine pairing. Patients suffering from inflammatory conditions such as atherosclerosis could benefit from these kinds of therapeutics.
\end{abstract}

View the article online

https://www.science.org/doi/10.1126/scitranslmed.aah6650

Permissions

https://www.science.org/help/reprints-and-permissions 